\title{
Algebraic description of chain transitivity for semigroup actions on flag bundles
}

\author{
Josiney A. Souza ${ }^{1}$
}

Received: 13 November 2017 / Accepted: 12 August 2018 / Published online: 23 August 2018

(c) Fondazione Annali di Matematica Pura ed Applicata and Springer-Verlag GmbH Germany, part of Springer Nature 2018

\begin{abstract}
This paper deals with the general concept of chain recurrence for semigroup actions in the special case of flag bundles. An algebraic description of the maximal chain transitive sets is presented in terms of singularities of one-parameter subgroups in the flag manifolds. This description yields a generalization of the Selgrade theorem for semigroup actions on projective bundles.
\end{abstract}

Keywords Semigroup actions $\cdot$ Vector bundle $\cdot$ Flag bundle $\cdot$ Chain recurrence $\cdot$ Morse decomposition

Mathematics Subject Classification 37B35 $\cdot 37 \mathrm{~B} 25 \cdot 54 \mathrm{H} 15 \cdot 54 \mathrm{H} 20$

\section{Introduction}

In this paper, we use the concept of fixed points of one-parameter subgroup to present the algebraic description of maximal chain transitive sets for semigroup actions on flag bundles. This description yields information about the maximal chain transitive sets, like their number and their ordering, and generalizes the Selgrade theorem for linear flows and linear semigroup actions on projective bundles [23,24].

In our setting, we start with a principal $G$-bundle $Q \rightarrow B$ with $G$ a noncompact semisimple (or more generally reductive) Lie group, and let $S$ be a semigroup of homeomorphisms of $Q$ which commutes with the right action of $G$. If $F=G / P$ is a flag manifold, where $P$ is a parabolic subgroup of $G$, we form the associated flag bundle $E=Q \times_{G} F \rightarrow B$. We are interested in the chain transitivity of the action of $S$ induced on $E$. Our main theorem shows that the maximal chain transitive sets are given fiberwise by the connected components of the fixed point set of $\exp (t H)$ for some $H$ in the Lie algebra of $G$. For instance, if $G$ is the reductive Lie group $\mathrm{Gl}(n, \mathbb{R})$ acting on the topological space $Q$, let $S \subset \mathbb{R}^{n}$ be a convex cone and define the action of $S$ on $Q$ by

$凶$ Josiney A. Souza

jasouza3@uem.br

1 Departamento de Matemática, Universidade Estadual de Maringá, Maringá, PR 87020-900, Brazil 


$$
\left(t_{1}, \ldots, t_{n}\right) q=q \operatorname{diag}\left\{\mathrm{e}^{t_{1}+\cdots+t_{n}}, \ldots, \mathrm{e}^{t_{1}+\cdots+t_{n}}\right\} .
$$

This action commutes with the right action of $\mathrm{Gl}(n, \mathbb{R})$. In another case, let $X_{1}, \ldots, X_{n}$ be pairwise commutative vector fields on a manifold $M$ and define the action of the cone $S \subset \mathbb{R}^{n}$ on $M$ by

$$
\left(t_{1}, \ldots, t_{n}\right) x=\exp \left(t_{1} X_{1}\right) \circ \cdots \circ \exp \left(t_{n} X_{n}\right)(x) .
$$

This action lifts to an action that commutes with the right action of $\mathrm{Gl}(n, \mathbb{R})$ on the bundle of frames BM (see the section of examples). More generally, let $\Sigma$ be a control system on $M$ determined by a set of vector fields $F$. This system defines a semigroup of diffeomorphisms

$$
S=\left\{\exp \left(t_{1} X_{1}\right) \circ \cdots \circ \exp \left(t_{k} X_{k}\right): X_{i} \in F, \sum_{i=1}^{k} t_{i} \geq 0, t_{i} \geq 0, k \in \mathbb{N}\right\}
$$

which describes its trajectories. This control system lifts to a linear control system $\mathrm{T} \Sigma$ on the tangent bundle $\mathrm{T} M \rightarrow M$, whose fibers are the tangent spaces $\mathrm{T}_{x} M$ and the structural group is $\mathrm{Gl}(n, \mathbb{R})$. The flag manifolds of $\mathrm{Gl}(n, \mathbb{R})$ are the flags of subspaces of $\mathbb{R}^{n}$, and the associated flag bundles are the bundles over $M$ which are built by taking flags of subspaces of $\mathrm{T}_{x} M$. The projective bundle $\mathbb{P}(\mathrm{T} M) \rightarrow M$ is a special case of flag bundle, where the fibers are the projective spaces $\mathbb{P}_{x}$ of $T_{x} M$. In this case, the fixed points of $\exp \left(t H_{x}\right)$ in $\mathbb{P}_{x}$ are the projection of the eigenvectors of $H_{x}$, and the connected components of the fixed point set are the projection of the eigenspaces of $H_{x}$. If the control system is chain transitive on $M$, the maximal chain transitive sets in $\mathbb{P}(\mathrm{T} M)$ are given by $\bigcup_{x \in M} \mathbb{P}\left(V_{\lambda_{i}}^{x}\right)$, where $\lambda_{1}>\lambda_{2}>\cdots>\lambda_{k}$ are the distinct eigenvalues of $H_{x}$ and $V_{\lambda_{i}}^{x}$ is the $\lambda_{i}$-eigenspace of $H_{x}$. This approaches the Selgrade theorem [24].

We follow the methodology of shadowing semigroup developed by Braga Barros and San Martin [4]. They have introduced the formal concept of chain recurrence for semigroup action that depends on a family of subsets of the semigroup. Their main theorem characterizes the chain control sets as intersections of control sets for the shadowing semigroups. The same methodology was used to give an algebraic description of the maximal chain transitive sets for flows and semiflows on flag bundles $[3,16]$.

The paper [24] contains previous results on chain transitivity for semigroup actions on flag bundles. If $\pi_{\mathbb{E}_{\Theta}}: \mathbb{E}_{\Theta}=Q \times_{G} \mathbb{F}_{\Theta} \rightarrow B$ is a flag bundle, associated with a locally trivial principal bundle $\pi: Q \rightarrow B$, and $S$ is a semigroup of automorphisms of $Q$ that is $\mathcal{F}$-chain transitive on the base space $B$, with $\mathcal{F}$ a filter basis satisfying some translations hypotheses, then the maximal $\mathcal{F}$-chain transitive sets of $S$ on $\mathbb{E}_{\Theta}$ are described as the sets

$$
E_{\Theta}(w)=\bigcap_{\mathcal{U}_{\varepsilon} \in \mathcal{O}_{\Theta}, A \in \mathcal{F}} \operatorname{cls}\left(\mathbb{D}_{\mathcal{U}_{\varepsilon}, A}^{\Theta}(w)_{0}\right),
$$

where $w$ is an element of the Weyl group $W$ of the semi-simple Lie group $G, \mathcal{O}_{\Theta}$ is a special admissible family of open covering of $\mathbb{E}_{\Theta}$, and $\mathbb{D}_{\mathcal{U}_{\varepsilon}, A}^{\Theta}(w)$ is the $w$-control set of the shadowing semigroup $\operatorname{End}_{l}\left(\mathbb{E}_{\Theta}\right)_{\mathcal{U}_{\varepsilon}, A}$ given by local endomorphisms. The intersections of $E_{\Theta}(w)$ with the fibers $\left(\mathbb{E}_{\Theta}\right)_{\pi(q)}=\pi_{\mathbb{E}_{\Theta}}^{-1}(\pi(q))$ are described by

$$
E_{\Theta}(1) \cap\left(\mathbb{E}_{\Theta}\right)_{\pi(q)}=q \cdot \bigcap_{\mathcal{U}_{\varepsilon}, A} \mathbb{A}_{\mathcal{U}_{\varepsilon}, A}^{\Theta}(1)_{0},
$$

where $\mathbb{A}_{\mathcal{U}_{\varepsilon}, A}^{\Theta}(1)_{0}$ is the transitivity set of the control set $\mathbb{A}_{\mathcal{U}_{\varepsilon}, A}^{\Theta}$ (1) for the shadowing semigroup induced on the flag $\mathbb{F}_{\Theta}$. From this characterization, we reproduce the strategies of 
$[3,16]$ to prove that $E_{\Theta}(1) \cap\left(\mathbb{E}_{\Theta}\right)_{\pi(q)}$ is an algebraic variety of fixed points for an element $h \in G$ (Theorem 4.1).

The Selgrade theorem for linear flows on vector bundles shows that the maximal chain transitive sets of a flow on the projective bundle which covers a chain transitive flow on the base space decompose the vector bundle in a Whitney sum [23]. The theorem includes the existence of a finest Morse decomposition in the projective bundle whose Morse sets coincide with the maximal chain transitive sets. It was generalized by Colonius and Kliemann [10] by showing the existence of a finest Morse decomposition in the bundles whose fibers are flag manifolds of subspaces of a vector bundle. Braga Barros and San Martin [3] extended the Colonius-Kliemann theorem to generalized flag bundles by showing that maximal chain transitive sets for an automorphism flow are intersections of control sets for shadowing semigroups [3, Theorem 4.7]. If the limit sets on the base space $B$ of the bundle are nonempty, it follows that there is an adjoint orbit $\operatorname{Ad}(G) H$ in the Lie algebra $\mathfrak{g}$ of $G$ and a map $q \in Q \mapsto h(q) \in \operatorname{Ad}(G) H$ such that the intersection of a maximal chain transitive set with the fiber over $x$ is given by the singularities of $h(q)$ [3, Theorem 9.11]. In the special case of projective bundle, it is possible to obtain on each fiber over $x$ a diagonalizable linear map $H_{x}$ whose eigenspaces are the maximal chain transitive sets, recovering the results of Selgrade and Colonius-Kliemann. It is interesting to observe that their method of proof does not require compactness of the base space, because the result is stated by means of maximal chain transitive sets, which in the compact case coincide with the finest Morse decomposition. More recently, the paper [24] extended the Selgrade theorem to linear semigroup actions on projective bundle, recovering the classical Selgrade's result but not recovering the result of Braga Barros-San Martin about generalized flag bundles. In the present paper, we extend the result of Braga Barros-San Martin to the general setting of semigroup of automorphisms on flag bundles (Theorem 5.1).

\section{Control sets in flag bundles}

This section contains the basic definitions and background results about semigroup of endomorphisms on flag bundles. We recall some concepts of the real semi-simple theory, including flag bundles and control sets, and prove a strategic lemma on fixed points of one-parameter subgroups. We refer to $[2,11,25,26]$ for the concepts of semi-simple Lie groups and flag manifolds and to $[13,14]$ for the notions of fiber bundles.

\subsection{Flag manifolds}

Let $G$ be a connected semi-simple Lie group with noncompact Lie algebra $\mathfrak{g}$. Take a Cartan decomposition $\mathfrak{g}=\mathfrak{k} \oplus \mathfrak{s}$ with corresponding Cartan involution $\theta$. This means that $\theta$ is an involutive automorphism of $\mathfrak{g}, \mathfrak{k}=\{X \in \mathfrak{g}: \theta(X)=X\}$, and $\mathfrak{s}=\{X \in \mathfrak{g}: \theta(X)=-X\}$. Let $\mathfrak{a} \subset \mathfrak{s}$ be a maximal abelian subalgebra and denote by $\Pi$ the set of roots of the pair $(\mathfrak{g}, \mathfrak{a})$, that is, the non-trivial linear functionals of $\mathfrak{a}$ such that $\alpha(H)$ is an eigenvalue of the adjoint map ad $(H)$, for all $\alpha \in \Pi$ and $H \in \mathfrak{a}$. For each $\alpha \in \Pi$, denote by $\mathfrak{g}_{\alpha}$ the $\alpha$-root space

$$
\mathfrak{g}_{\alpha}=\{X \in \mathfrak{g}: \operatorname{ad}(H)(X)=\alpha(H)(X) \text { for every } H \in \mathfrak{a}\} .
$$

We have

$$
\mathfrak{g}=\mathfrak{m} \oplus \mathfrak{a} \oplus \sum_{\alpha \in \Pi} \mathfrak{g}_{\alpha}
$$


where $\mathfrak{m}=\left\{X \in \mathfrak{k}:\right.$ ad $\left.\left.(X)\right|_{\mathfrak{a}}=0\right\}$ is the centralizer of $\mathfrak{a}$ in $\mathfrak{k}$.

The Weyl chambers associated with the pair $(\theta, \mathfrak{a})$ are the connected components of the set

$$
\{H \in \mathfrak{a}: \alpha(H) \neq 0 \text { for all } \alpha \in \Pi\} .
$$

By choosing a Weyl chamber $\mathfrak{a}^{+} \subset \mathfrak{a}$, consider the corresponding set of positive roots

$$
\Pi^{+}=\left\{\alpha \in \Pi: \alpha(H)>0 \text { for all } H \in \mathfrak{a}^{+}\right\}
$$

and take the subalgebras

$$
\mathfrak{n}^{+}=\sum_{\alpha \in \Pi^{+}} \mathfrak{g}_{\alpha} \quad \mathfrak{n}^{-}=\sum_{\alpha \in \Pi^{+}} \mathfrak{g}_{-\alpha} .
$$

Then, $\mathfrak{n}^{+}$is nilpotent, $\mathfrak{n}^{-}=\theta\left(\mathfrak{n}^{+}\right)$since $\theta\left(\mathfrak{g}_{\alpha}\right)=\mathfrak{g}_{-\alpha}$, and

$$
\mathfrak{g}=\mathfrak{k} \oplus \mathfrak{a} \oplus \mathfrak{n}^{+}
$$

is the Iwasawa decomposition of $\mathfrak{g}$ associated with the admissible triple $\left(\theta, \mathfrak{a}, \mathfrak{a}^{+}\right)$.

The simple system of roots associated with $\left(\theta, \mathfrak{a}, \mathfrak{a}^{+}\right)$is the set $\Sigma$ of every positive root that cannot be described as a linear combination of two roots. For a given subset $\Theta \subset \Sigma$ denote by $\langle\Theta\rangle$ the subset of $\Pi$ spanned by $\Theta$ over the integers. Denote $\langle\Theta\rangle^{+}=\langle\Theta\rangle \cap \Pi^{+}$ and define the subalgebras

$$
\mathfrak{n}^{+}(\Theta)=\sum_{\alpha \in\langle\Theta\rangle^{+}} \mathfrak{g}_{\alpha} \quad \mathfrak{n}^{-}(\Theta)=\sum_{\alpha \in\langle\Theta\rangle^{+}} \mathfrak{g}_{-\alpha} .
$$

The standard minimal parabolic subalgebra is defined by $\mathfrak{p}=\mathfrak{m} \oplus \mathfrak{a} \oplus \mathfrak{n}^{+}$, and the standard parabolic subalgebra $\mathfrak{p}_{\Theta}$ of type $\Theta$ is given by $\mathfrak{p}_{\Theta}=\mathfrak{n}^{-}(\Theta) \oplus \mathfrak{p}$. The standard parabolic subgroup $P_{\Theta}$ of $G$ is defined as the normalizer of $\mathfrak{p}_{\Theta}$ in $G$

$$
P_{\Theta}=\left\{g \in G: \operatorname{Ad}(g) \mathfrak{p}_{\Theta}=\mathfrak{p}_{\Theta}\right\},
$$

where Ad $: G \rightarrow \mathrm{Gl}(\mathfrak{g})$ is the adjoint representation. The homogeneous space $\mathbb{F}_{\Theta}=G / P_{\Theta}$ is the associated flag manifold of type $\Theta$. It is a compact metrizable space and identifies with the set of all parabolic subalgebras of type $\Theta$. For two subsets $\Delta \subset \Theta \subset \Sigma$, we have the standard fibration $\pi_{\Theta}^{\Delta}: \mathbb{F}_{\Delta} \rightarrow \mathbb{F}_{\Theta}$ whose fiber is $P_{\Theta} / P_{\Delta}$. We write simply $\mathbb{F}$ for the maximal flag manifold $\mathbb{F}_{\emptyset}$ and $\pi_{\Theta}$ for the standard fibration $\pi_{\Theta}^{\emptyset}$.

By denoting $K=\langle\exp (\mathfrak{k})\rangle, A=\langle\exp (\mathfrak{a})\rangle$, and $N^{ \pm}=\exp \left(\mathfrak{n}^{ \pm}\right)$the connected subgroups of $G$ with corresponding Lie algebras, one has the Iwasawa decomposition of $G$ given by $G=K A N^{+}$. The group $K$ acts transitively on each flag manifold $\mathbb{F}_{\Theta}$, allowing an identification $\mathbb{F}_{\Theta}=K / K_{\Theta}$ where $K_{\Theta}=K \cap P_{\Theta}$. Let $H_{\Theta} \in \operatorname{cls}\left(\mathfrak{a}^{+}\right)$be such that $\Theta=$ $\left\{\alpha \in \Sigma: \alpha\left(H_{\Theta}\right)=0\right\}$. Then, $K_{\Theta}$ is the centralizer of $H_{\Theta}$ in $K$ so that the adjoint orbit Ad $(K) H_{\Theta}$ identifies with $\mathbb{F}_{\Theta}$. Conversely, for a given $H \in \operatorname{cls}\left(\mathfrak{a}^{+}\right)$, Ad $(K) H$ identifies with $\mathbb{F}_{\Theta(H)}$ where $\Theta(H)=\{\alpha \in \Sigma: \alpha(H)=0\}$.

\subsection{Fixed points of one-parameter subgroups}

The Weyl group $W$ of $\mathfrak{g}$ is the group generated by the reflections with respect to roots in $\Pi$. This group is isomorphic with $M^{*} / M$, where $M^{*}$ and $M$ are the normalizer and centralizer of $\mathfrak{a}$ in $K$, respectively. The orbit of $H_{\Theta}$ under $M^{*}$ is finite and the action of $M^{*}$ on this orbit factors through $M$. Thus, $W$ is a finite group. Denote $b_{\Theta}^{+}$the origin of $\mathbb{F}_{\Theta}$, that is, the point 
which identifies with $H_{\Theta}$. Then, the orbit of $H_{\Theta}$ identifies with the set $\left\{w b_{\Theta}^{+}: w \in W\right\}$. The principal involution of $W$ is the unique element $w_{0} \in W$ such that $w_{0} \Sigma=-\Sigma$.

For a given $H \in \operatorname{cls}\left(\mathfrak{a}^{+}\right)$, the set of fixed points of the adjoint action of the one-parameter subgroup $\exp (t H)$ on the flag manifold $\mathbb{F}_{\Theta}$ is described by the disjoint reunion of connected subsets

$$
\bigcup_{w \in W_{H} \backslash W} K_{H}^{0} w b_{\Theta}^{+},
$$

where $W_{H} \subset W$ is the stabilizer of $H$ and $K_{H}^{0}$ is the connected component of the identity of the centralizer $K_{H}$ of $H$ in $K$ [11, Proposition 1.3 and Corollary 3.5]. Denote fix $\Theta(H, w)=$ $K_{H}^{0} w b_{\Theta}^{+}$for each $H \in \operatorname{cls}\left(\mathfrak{a}^{+}\right)$and $w \in W$. If $H \in \mathfrak{a}^{+}(H$ is regular $)$, then $K_{H}^{0}=M_{0}$ is the connected component of the identity of $M$; hence, $\operatorname{fix}_{\Theta}(H, w)=\left\{w b_{\Theta}^{+}\right\}$for every $w \in W$. In this case, by denoting $h=\exp H$, we have $h^{n} x \rightarrow w b_{\Theta}^{+}$, as $n \rightarrow+\infty$, for every $x \in N^{-} w b_{\Theta}^{+}$, and therefore the orbit $N^{-} w b_{\Theta}^{+}$is the stable manifold, while the orbit $N^{+} w b_{\Theta}^{+}$is the unstable manifold of the fixed point $w b_{\Theta}^{+}$. Thus, there is a unique attractor $b_{\Theta}^{+}$, whose stable manifold is the open and dense orbit $N^{-} b_{\Theta}^{+}$, and there is a unique repeller $w_{0} b_{\Theta}^{+}$, whose unstable manifold is the open and dense orbit $N^{+} w_{0} b_{\Theta}^{+}$. In general, if $H^{\prime}$ is split-regular, that is, $H^{\prime}=\operatorname{Ad}(g)(H)$ for some $g \in G$ and $H \in \mathfrak{a}^{+}$, then $\operatorname{fix}_{\Theta}\left(H^{\prime}, w\right)=$ $g \operatorname{fix}_{\Theta}(H, w)=\left\{g w b_{\Theta}^{+}\right\}$, with stable manifold $g N^{-} w b_{\Theta}^{+}$and unstable manifold $g N^{+} w b_{\Theta}^{+}$. We also say that an element $h \in G$ is split-regular if $h=\exp (\operatorname{Ad}(g)(H))=g \exp (H) g^{-1}$ for some $g \in G$ and $H \in \mathfrak{a}^{+}$, and we denote $\operatorname{fix}_{\Theta}(h, w)=g \operatorname{fix}_{\Theta}(H, w)=\left\{g w b_{\Theta}^{+}\right\}$, $\operatorname{st}_{\Theta}(h)=g N^{-} b_{\Theta}^{+}$, and un $\Theta(h)=g N^{+} b_{\Theta}^{+}$.

Take $H_{\Theta} \in \operatorname{cls}\left(\mathfrak{a}^{+}\right)$such that $\Theta=\Theta\left(H_{\Theta}\right)=\left\{\alpha \in \Sigma: \alpha\left(H_{\Theta}\right)=0\right\}$. Define $\mathfrak{p}_{\Theta}^{-}=\mathfrak{n}^{+}(\Theta) \oplus \mathfrak{p}^{-}$where $\mathfrak{p}^{-}=\mathfrak{m} \oplus \mathfrak{a} \oplus \mathfrak{n}^{-}$. We have $\theta\left(\mathfrak{p}_{\Theta}\right)=\mathfrak{p}_{\Theta}^{-}$. The subgroup of $G$ that fixes both $\mathfrak{p}_{\Theta}$ and $\mathfrak{p}_{\Theta}^{-}$, or in other words, the isotropy subgroup of $\left(\mathfrak{p}_{\Theta}, \mathfrak{p}_{\Theta}^{-}\right)$, is the intersection of the normalizers $N_{G}\left(\mathfrak{p}_{\Theta}\right)$ and $N_{G}\left(\mathfrak{p}_{\Theta}^{-},\right)$of $\mathfrak{p}_{\Theta}$ and $\mathfrak{p}_{\Theta}^{-}$in $G$, respectively, which coincides with the centralizer $Z_{G}\left(H_{\Theta}\right)$ of $H_{\Theta}$ in $G$ [3, p. 24]. Then, we have $\operatorname{fix}_{\Theta}\left(H_{\Theta}, 1\right)=\left\{b_{\Theta}^{+}\right\}$and fix $\Theta^{*}\left(H_{\Theta}, w_{0}\right)=\left\{w_{0} b_{\Theta *}^{+}\right\}$, where $\Theta^{*}=-w_{0} \Theta$ is the dual of $\Theta$. In fact, since fix $x_{\Theta}\left(H_{\Theta}, 1\right)=K_{H_{\Theta}}^{0} b_{\Theta}^{+}$and $K_{H_{\Theta}} \subset Z_{G}\left(H_{\Theta}\right)=N_{G}\left(\mathfrak{p}_{\Theta}\right) \cap N_{G}\left(\mathfrak{p}_{\Theta}^{-},\right)$, we have $\operatorname{fix}_{\Theta}\left(H_{\Theta}, 1\right)=\left\{b_{\Theta}^{+}\right\}$. In the other case, we have $w_{0} \mathfrak{p}_{\Theta}^{-}=w_{0} \theta\left(\mathfrak{p}_{\Theta}\right)=\mathfrak{p}_{\Theta *}[19$, Lemma 2.2], and then $\mathfrak{p}_{\Theta}^{-}=w_{0} \mathfrak{p}_{\Theta^{*}}$. Hence, fix $\Theta_{\Theta^{*}}\left(H_{\Theta}, w_{0}\right)=K_{H_{\Theta}}^{0} w_{0} b_{\Theta^{*}}^{+}=\left\{w_{0} b_{\Theta^{*}}^{+}\right\}$.

For the following, denote by $b^{+}$the origin of the maximal flag manifold $\mathbb{F}$.

Lemma 2.1 For a given $\Theta \subset \Sigma$, let $H_{\Theta} \in \operatorname{cls}\left(\mathfrak{a}^{+}\right)$such that $\Theta=\Theta\left(H_{\Theta}\right)$. Then,

$$
\text { fix }\left(H_{\Theta}, 1\right)=\pi_{\Theta}^{-1}\left(\operatorname{fix}_{\Theta}\left(H_{\Theta}, 1\right)\right) \quad \text { and } \quad \operatorname{fix}\left(H_{\Theta}, w_{0}\right)=\pi_{\Theta^{*}}^{-1}\left(\operatorname{fix}_{\Theta^{*}}\left(H_{\Theta}, w_{0}\right)\right) .
$$

Proof For $H \in \operatorname{Ad}(G) H_{\Theta}$, we have $\pi_{\Theta}\left(K_{H}^{0} w b^{+}\right)=K_{H}^{0} w b_{\Theta}^{+}$for every $w \in W$, since the standard fibration $\pi_{\Theta}: \mathbb{F} \rightarrow \mathbb{F}_{\Theta}$ is equivariant. Hence, $\pi_{\Theta}(\operatorname{fix}(H, w))=\operatorname{fix}_{\Theta}(H, w)$ for every $w \in W$. Writing $H=g H_{\Theta}$, it follows that

$$
\pi_{\Theta}(\operatorname{fix}(H, 1))=\left\{g b_{\Theta}^{+}\right\} \quad \text { and } \quad \pi_{\Theta *}\left(\operatorname{fix}\left(H, w_{0}\right)\right)=\left\{g w_{0} b_{\Theta^{*}}^{+}\right\} .
$$

Then, we have fix $\left(H_{\Theta}, 1\right) \subset \pi_{\Theta}^{-1}\left(\operatorname{fix}_{\Theta}\left(H_{\Theta}, 1\right)\right)$ and fix $\left(H_{\Theta}, w_{0}\right) \subset \pi_{\Theta *}^{-1}\left(\right.$ fix $\left.\Theta^{*}\left(H_{\Theta}, w_{0}\right)\right)$. On the other hand, for a given $k b_{\Theta}^{+} \in \pi_{\Theta}^{-1}\left(\right.$ fix $\left.x_{\Theta}\left(H_{\Theta}, 1\right)\right)$, with $k \in K$, we have $k b_{\Theta}^{+}=b_{\Theta}^{+}$, and then $k \in K \cap P_{\Theta}=K_{\Theta}$. Hence, $\operatorname{Ad}(k) H_{\Theta}=H_{\Theta}$ and therefore

$$
\begin{aligned}
\operatorname{Ad}\left(\exp t H_{\Theta}\right) \operatorname{Ad}(k) b^{+} & =\operatorname{Ad}(k) \operatorname{Ad}\left(\exp \left(\operatorname{Ad}\left(k^{-1}\right)\left(t H_{\Theta}\right)\right)\right) b^{+} \\
& =\operatorname{Ad}(k) \operatorname{Ad}\left(\exp t H_{\Theta}\right) b^{+} \\
& =\operatorname{Ad}(k) b^{+} .
\end{aligned}
$$


This means that $k b^{+}$is fixed by $\exp \left(t H_{\Theta}\right)$, and thus $k b^{+} \in$ fix $\left(H_{\Theta}, w\right)$ for some $w \in W$. Hence, $\operatorname{fix}_{\Theta}\left(H_{\Theta}, w\right)=\operatorname{fix}_{\Theta}\left(H_{\Theta}, 1\right)$, that is, $w$ and 1 represent the same equivalent class in $W_{H_{\Theta}} \backslash W$. Thus, fix $\left(H_{\Theta}, w\right)=$ fix $(H, 1)$, and since $K$ acts transitively on $\mathbb{F}$, we have $\pi_{\Theta}^{-1}\left(\operatorname{fix}_{\Theta}\left(H_{\Theta}, 1\right)\right) \subset$ fix $\left(H_{\Theta}, 1\right)$, and therefore fix $\left(H_{\Theta}, 1\right)=\pi_{\Theta}^{-1}\left(\right.$ fix $\left.x_{\Theta}\left(H_{\Theta}, 1\right)\right)$. Now, take $H_{\Theta^{*}} \in \operatorname{cls}\left(\mathfrak{a}^{+}\right)$such that $\Theta^{*}=\Theta\left(H_{\Theta^{*}}\right)$. As $\Theta^{*}=-w_{0} \Theta$, it is easily seen that $\Theta\left(H_{\Theta}\right)=\Theta\left(w_{0} H_{\Theta *}\right)$, and then $K_{H_{\Theta}}=K_{w_{0} H_{\Theta *}}$. Hence, for a given $k b^{+} \in$ $\pi_{\Theta^{*}}^{-1}\left(\right.$ fix $\left._{\Theta^{*}}\left(H_{\Theta}, w_{0}\right)\right)$, with $k \in K$, we have $k b_{\Theta^{*}}^{+}=w_{0} b_{\Theta^{*}}^{+}$, that is, $k w_{0} w_{0} b_{\Theta^{*}}^{+}=w_{0} b_{\Theta^{*}}^{+}$. Identifying $w_{0}$ with $k_{0} M$, for some $k_{0} \in M^{*}$, we have

$$
k k_{0} \in k_{0} K_{H_{\Theta}}=K_{w_{0} H_{\Theta *}}=K_{H_{\Theta}} .
$$

Hence, $k b^{+}=k w_{0} w_{0} b^{+} \in K_{H_{\Theta}} w_{0} b^{+}$, and thus $k b^{+} \in$ fix $\left(H_{\Theta}, w_{0}\right)$. This means that $\pi_{\Theta^{*}}^{-1}\left(\operatorname{fix}_{\Theta^{*}}\left(H_{\Theta}, w_{0}\right)\right) \subset$ fix $\left(H_{\Theta}, w_{0}\right)$, and therefore fix $\left(H_{\Theta}, w_{0}\right)=\pi_{\Theta^{*}}^{-1}\left(\operatorname{fix}_{\Theta *}\left(H_{\Theta}, w_{0}\right)\right)$.

Corollary 2.1 Let $H \in \operatorname{Ad}(G) H_{\Theta}$, with $H_{\Theta} \in \operatorname{cls}\left(\mathfrak{a}^{+}\right)$such that $\Theta=\Theta\left(H_{\Theta}\right)$. Then,

$$
\operatorname{fix}(H, 1)=\pi_{\Theta}^{-1}\left(\operatorname{fix}_{\Theta}(H, 1)\right) \quad \text { and } \quad \operatorname{fix}\left(H, w_{0}\right)=\pi_{\Theta^{*}}^{-1}\left(\operatorname{fix}_{\Theta^{*}}\left(H, w_{0}\right)\right) .
$$

Proof By using Lemma 2.1, it is enough to show that fix $\mathrm{x}_{\Theta}(g H, w)=g \mathrm{fix}_{\Theta}(H, w)$ for all $H \in \operatorname{cls}\left(\mathfrak{a}^{+}\right), g \in G$, and $w \in W$. Indeed, for $x \in \operatorname{fix}_{\Theta}(H, w)$, we have

$$
\begin{aligned}
\operatorname{Ad}(\exp t(g H)) \operatorname{Ad}(g)(x) & =\operatorname{Ad}(\exp (\operatorname{Ad}(g) t H)) \operatorname{Ad}(g)(x) \\
& =\operatorname{Ad}\left(g \exp (t H) g^{-1}\right) \operatorname{Ad}(g)(x) \\
& =\operatorname{Ad}(g) \operatorname{Ad}(\exp (t H))(x) \\
& =\operatorname{Ad}(g)(x) .
\end{aligned}
$$

Hence, $g \operatorname{fix}_{\Theta}(H, w) \subset \operatorname{fix}_{\Theta}(g H, w)$. The equality follows by the symmetry.

\subsection{Flag bundles and control sets}

Consider a right action $(q, g) \in Q \times G \rightarrow q g \in Q$ of $G$ on a topological space $Q$. Then, $G$ acts on the right on the product space $Q \times \mathbb{F}_{\Theta}$ by the map

$$
(q, v, g) \in Q \times \mathbb{F}_{\Theta} \times G \rightarrow(q, v) g=\left(q g, g^{-1} v\right) .
$$

We denote by $\mathbb{E}_{\Theta}$ the quotient space $Q \times{ }_{G} \mathbb{F}_{\Theta}$ and by $q \cdot v$ an element of $\mathbb{E}_{\Theta}$. A principal bundle is a quadruplet $(Q, \pi, B, G)$ where $\pi: Q \rightarrow B$ is an open and surjective map such that $\pi(q g)=\pi(q)$ for all $q \in Q$ and $g \in G$. A flag bundle of type $\Theta$ associated with $\pi$ is the fiber bundle $\pi_{\mathbb{E}_{\Theta}}: \mathbb{E}_{\Theta} \rightarrow B$ given by $\pi_{\mathbb{E}_{\Theta}}(q \cdot v)=\pi(q)$. Each fiber $\left(\mathbb{E}_{\Theta}\right)_{x}=\pi_{\mathbb{E}_{\Theta}}^{-1}(x)$ of the flag bundle is homeomorphic with the flag manifold $\mathbb{F}_{\Theta}$. If $\mathbb{F}=\mathbb{F}_{\emptyset}$ is the maximal flag manifold of $\mathfrak{g}$, we denote $\mathbb{E}=Q \times_{G} \mathbb{F}$ and $\pi_{\mathbb{E}}: \mathbb{E} \rightarrow B$ is called the maximal flag bundle. The standard fibration $\tilde{\pi}_{\Theta}: \mathbb{E} \rightarrow \mathbb{E}_{\Theta}$ of the flag bundles is given by

$$
\tilde{\pi}_{\Theta}(q \cdot v)=q \cdot \pi_{\Theta}(v),
$$

where $\pi_{\Theta}: \mathbb{F} \rightarrow \mathbb{F}_{\Theta}$ is the standard fibration of the flag manifolds.

Definition 2.1 Let $(Q, \pi, B, G)$ be a principal bundle. A local endomorphism of $Q$ is a map $\phi: \operatorname{dom} \phi \rightarrow Q$ such that

1. $\operatorname{dom} \phi=\pi^{-1}(U)$ where $U \subset B$ is an open set; 
2. $\phi(q g)=\phi(q) g$ for every $q \in \operatorname{dom} \phi$ and $g \in G$.

We denote by $\operatorname{End}_{l}(Q)$ the set of all local endomorphism of $Q$. If $\phi, \psi \in \operatorname{End}_{l}(Q)$ and $\phi^{-1}(\operatorname{dom} \psi) \neq \varnothing$, then the composition $\psi \circ \phi: \phi^{-1}(\operatorname{dom} \psi) \rightarrow Q$ is also a local endomorphism of $Q$. Thus, $\operatorname{End}_{l}(Q)$ is a local semigroup on $Q$. A local endomorphism $\phi \in \operatorname{End}_{l}(Q)$ maps fibers into fibers and hence induces a map from $\pi(\operatorname{dom} \phi)$ into $B$ given by $\phi_{B}(\pi(q))=\pi(\phi(q))$. For $\Theta \subset \Sigma$, we have the map $\phi_{\mathbb{E}_{\Theta}}(q \cdot v)=\phi(q) \cdot v$ whose domain is the open set in $\mathbb{E}_{\Theta}$ over $\pi(\operatorname{dom} \phi)$. We denote by $\operatorname{End}_{l}\left(\mathbb{E}_{\Theta}\right)$ the local semigroup in $\mathbb{E}_{\Theta}$ induced by $\operatorname{End}_{l}(Q)$.

Now, let $\mathcal{S} \subset \operatorname{End}_{l}(Q)$ be a local subsemigroup. It induces the semigroup $\mathcal{S}_{B}=$ $\left\{\phi_{B}: \phi \in \mathcal{S}\right\}$ on $B$ and the semigroup $\mathcal{S}_{\mathbb{E}_{\Theta}}=\left\{\phi_{\mathbb{E}_{\Theta}}: \phi \in \mathcal{S}\right\}$ on $\mathbb{E}_{\Theta}$. For $q \in Q$, we also define the set

$$
\mathcal{S}_{q}=\{g \in G: \text { there is } \phi \in \mathcal{S} \text { such that } \phi(q)=q g\}
$$

that is a subsemigroup of $G$ if it is nonempty. Note that $\mathcal{S}_{q}$ is open in $G$ if the orbit $\mathcal{S} q$ is open in $Q$. For others properties of the semigroup $\mathcal{S}_{q}$, we refer to [5].

We denote the forward and backward orbits of a local semigroup $\mathcal{S}$ over $q \in Q$, respectively, by

$$
\begin{aligned}
\mathcal{S} q & =\{\phi(q): \phi \in \mathcal{S}, q \in \operatorname{dom} \phi\}, \\
\mathcal{S}^{*} q & =\{x \in Q: \text { there is } \phi \in \mathcal{S} \text { with } \phi(x)=q\} .
\end{aligned}
$$

Definition 2.2 A nonempty set $D \subset Q$ is said to be a control set for the local semigroup $\mathcal{S}$ if

1. int $(D) \neq \varnothing$,

2. $D \subset \operatorname{cls}(\mathcal{S} x)$ for all $x \in D$,

3. $D$ is maximal satisfying these properties.

The open set $D_{0}=\left\{x \in D: x \in \operatorname{int}(\mathcal{S} x) \cap \operatorname{int}\left(\mathcal{S}^{*} x\right)\right\}$ is called the transitivity set of the control set $D$. When $D_{0}$ is nonempty, $D$ is called an effective control set for $\mathcal{S}$. In this case, $D_{0}$ is a dense subset in $D$. The control set $D$ is called invariant if cls $(D)=\operatorname{cls}(\mathcal{S} x)$ for all $x \in D$. Then, $D$ is a closed invariant control set of $\mathcal{S}$ if and only if it is a minimal set of $\mathcal{S}$.

The domain of attraction of a control set $D$ of $\mathcal{S}$ is defined as

$$
\mathcal{A}(D)=\{q \in Q: \operatorname{cls}(\mathcal{S} q) \cap D \neq \emptyset\} .
$$

If $D$ is an effective control set, we have

$$
\mathcal{A}(D)=\left\{q \in Q: \mathcal{S} q \cap D_{0} \neq \emptyset\right\} .
$$

In this case, we define the domain of repellency of $D$ by

$$
\mathcal{A}^{*}(D)=\left\{q \in Q: \mathcal{S}^{*} q \cap D_{0} \neq \emptyset\right\} .
$$

The dynamic ordering among the control sets is given as the following: For two control sets $D$ and $D^{\prime}, D \preceq D^{\prime}$ means $D \cap \mathcal{A}\left(D^{\prime}\right) \neq \emptyset$, that is, $D^{\prime}$ attracts $D$. The invariant control sets are maximal with respect to the dynamic ordering.

Assume that the local semigroup $\mathcal{S} \subset \operatorname{End}_{l}(Q)$ is transitive in $B$ and its forward and backward orbits in $Q$ are open sets. For each $q \in Q$, the subsemigroup $\mathcal{S}_{q} \subset G$ is open [16, Lemma 4.16]. In [21], the effective control sets for the action of $\mathcal{S}_{q}$ on the maximal flag manifold were described by means of the Weyl group $W$ of $\mathfrak{g}$. In this description, we have a mapping

$$
w \rightarrow \mathbb{A}^{q}(w)
$$


which associates with $w \in W$ a control set $\mathbb{A}^{q}(w)$ in such a way that the transitivity set $\mathbb{A}^{q}(w)_{0}$ is the set of the fixed points of type $w$ for the split-regular elements in int $\left(\mathcal{S}_{q}\right)$. The set $\mathbb{A}^{q}(1)$ is the unique invariant control set and it is closed. This means that $\mathbb{A}^{q}(1)$ is $\mathcal{S}_{q}$-invariant. Moreover, its transitivity set $\mathbb{A}^{q}(1)_{0}$ coincides with the set of attractors for the split-regular elements in int $\left(\mathcal{S}_{q}\right)$. On the other hand, the set $\mathbb{A}^{q}\left(w_{0}\right)$ is the unique minimal control set and $\mathbb{A}^{q}\left(w_{0}\right)_{0}$ coincides with the set of repellers for the split-regular elements in int $\left(\mathcal{S}_{q}\right)$. Similarly, for the inverted semigroup $\mathcal{S}_{q}^{-1}=\left\{g^{-1}: g \in \mathcal{S}_{q}\right\}$, one has a mapping $w \rightarrow \mathbb{A}^{q}(w)^{-}$into the control sets of $\mathcal{S}_{q}^{-1}$. The relation between the control sets of $\mathcal{S}_{q}$ and the control sets of $\mathcal{S}_{q}^{-1}$ is given by $\mathbb{A}^{q}(w)_{0}^{-}=\mathbb{A}^{q}\left(w_{0} w\right)_{0}$ [18, Proposition 3.1].

For each $\Theta \subset \Sigma$, we denote $\mathbb{A}_{\Theta}^{q}(w)$ the effective control set of $\mathcal{S}_{q}$ in $\mathbb{F}_{\Theta}$ such that $\pi_{\Theta}\left(\mathbb{A}^{q}(w)\right) \subset \mathbb{A}_{\Theta}^{q}(w)$ and $\pi_{\Theta}\left(\mathbb{A}^{q}(w)_{0}\right)=\mathbb{A}_{\Theta}^{q}(w)_{0}$. There is an effective control set $\mathbb{D}_{\Theta}^{q}(w)$ of $\mathcal{S}_{\mathbb{E}_{\Theta}}$ such that $\mathbb{D}_{\Theta}^{q}(w)_{0} \cap\left(\mathbb{E}_{\Theta}\right)_{\pi(q)} \neq \emptyset$ and

$$
\mathbb{D}_{\Theta}^{q}(w)_{0} \cap\left(\mathbb{E}_{\Theta}\right)_{\pi(q)}=q \cdot \mathbb{A}_{\Theta}^{q}(w)_{0} .
$$

These are all the effective control sets of $\mathcal{S}_{\mathbb{E}_{\Theta}}$ whose transitivity sets intersect the fiber $\left(\mathbb{E}_{\Theta}\right)_{\pi(q)}$. Since $\mathcal{S}$ is transitive in $B$, the transitivity set of an effective control set $\mathbb{D}$ of $\mathcal{S}_{\mathbb{E}_{\Theta}}$ is projected onto the base space, that is, $\pi_{\mathbb{E}_{\Theta}}\left(\mathbb{D}_{0}\right)=B$. Hence, $\mathbb{D}_{0}$ intersect the fiber $\left(\mathbb{E}_{\Theta}\right)_{\pi(q)}$. Furthermore, the control set $\mathbb{D}_{\Theta}^{q}(w)$ does not depend on $q \in Q$ [16, Theorem 5.8]. Therefore, $\left\{\mathbb{D}_{\Theta}^{q}(w): w \in W\right\}$ is the collection of all effective control sets of $\mathcal{S}_{\mathbb{E}_{\Theta}}$.

Lemma 2.2 Let $\mathcal{S}$ be a semigroup of local endomorphisms on $Q$. For a given control set $\mathbb{D}(w)$ of $\mathcal{S}_{\mathbb{E}}$ on $\mathbb{E}$, one has

$$
\begin{gathered}
\mathcal{A}(\mathbb{D}(w)) \cap \mathbb{E}_{\pi(q)}=q \cdot \mathcal{A}\left(\mathbb{A}^{q}(w)\right), \\
\mathcal{A}^{*}(\mathbb{D}(w)) \cap \mathbb{E}_{\pi(q)}=q \cdot \mathcal{A}^{*}\left(\mathbb{A}^{q}(w)\right),
\end{gathered}
$$

for every $q \in Q$, where $\mathbb{A}^{q}(w)$ is the effective $w$-control set of $\mathcal{S}_{q}$ on $\mathbb{F}$.

Proof Note that

$$
\begin{aligned}
\mathcal{A}\left(\mathbb{A}^{q}(w)\right) & =\left\{u \in \mathbb{F}: \mathcal{S}_{q} u \cap \mathbb{A}^{q}(w)_{0} \neq \emptyset\right\}, \\
\mathcal{A}^{*}\left(\mathbb{A}^{q}(w)\right) & =\left\{u \in \mathbb{F}: \mathcal{S}_{q}^{-1} u \cap \mathbb{A}^{q}(w)_{0} \neq \emptyset\right\} .
\end{aligned}
$$

If $q \cdot u \in \mathcal{A}(\mathbb{D}(w)) \cap \mathbb{E}_{\pi(q)}$, then there is $\phi \in \mathcal{S}$ such that $\phi(q) \cdot u \in \mathbb{D}(w)_{0}$. By taking $q \cdot v \in \mathbb{D}(w)_{0} \cap \mathbb{E}_{\pi(q)}=q \cdot \mathbb{A}(w)_{0}$, there is $\varphi \in \mathcal{S}$ such that $\varphi \phi(q) \cdot u=q \cdot v$. Hence, there is $g \in G$ with $\varphi \phi(q)=q g$ and $u=g^{-1} v$. This means that $g \in \mathcal{S}_{q}$, and then $u \in \mathcal{A}\left(\mathbb{A}^{q}(w)\right)$ since $v \in \mathbb{A}(w)_{0}$. Thus, $\mathcal{A}(\mathbb{D}(w)) \cap \mathbb{E}_{\pi(q)} \subset q \cdot \mathcal{A}\left(\mathbb{A}^{q}(w)\right)$. On the other hand, if $q \cdot u \in q \cdot \mathcal{A}\left(\mathbb{A}^{q}(w)\right)$, then there is $h \in \mathcal{S}_{q}$ such that $h u \in \mathbb{A}^{q}(w)_{0}$. Hence, there is $\phi \in \mathcal{S}$ such that $\phi(q) \cdot u=q \cdot h u \in q \cdot \mathbb{A}^{q}(w)_{0}=\mathbb{D}(w)_{0} \cap \mathbb{E}_{\pi(q)}$, which means that $q \cdot u \in \mathcal{A}(\mathbb{D}(w)) \cap \mathbb{E}_{\pi(q)}$. Therefore, $q \cdot \mathcal{A}\left(\mathbb{A}^{q}(w)\right)=\mathcal{A}(\mathbb{D}(w)) \cap \mathbb{E}_{\pi(q)}$. The proof for the second equality follows by similar way.

\section{Chain recurrence in flag bundles}

In this section, we present the previous results on chain recurrence for semigroup actions on flag bundles. We define the parabolic type of the semigroup action and show how the Weyl group plays a role in ordering the maximal chain transitive sets in a flag bundle. We refer to $[16,24]$ for details. 
Let $(Q, \pi, B, G)$ be a principal bundle that is locally trivial and the base space $B$ is paracompact. Consider a trivializing covering $\left\{U_{i}\right\}_{i \in I}$ of $Q$. For each $i \in I$, there is a homeomorphism $\psi_{i}: \pi^{-1}\left(U_{i}\right) \rightarrow U_{i} \times G, \psi_{i}=\left(\pi, v_{i}\right)$, where $v_{i}: \pi^{-1}\left(U_{i}\right) \rightarrow G$ is a continuous mapping such that $v_{i}(q g)=v_{i}(q) g$, for all $q \in \pi^{-1}\left(U_{i}\right)$ and $g \in G$. The family $\Psi=\left\{\left(U_{i}, \psi_{i}\right)\right\}_{i \in I}$ is called atlas of $Q$. For each $i \in I$, the application $v_{i}^{\mathbb{E}_{\Theta}}: \pi_{\mathbb{E}_{\Theta}}^{-1}\left(U_{i}\right) \rightarrow \mathbb{F}_{\Theta}$ given by $v_{i}^{\mathbb{E}_{\Theta}}(q \cdot u)=v_{i}(q) u$ is open. Hence, $\psi_{i}^{\mathbb{E}_{\Theta}}: \pi_{\mathbb{E}_{\Theta}}^{-1}\left(U_{i}\right) \rightarrow U_{i} \times \mathbb{F}_{\Theta}$, given by $\psi_{i}^{\mathbb{E}_{\Theta}}=\left(\pi_{\mathbb{E}_{\Theta}}, v_{i}^{\mathbb{E}_{\Theta}}\right)$, is a homeomorphism. Then, the family $\Psi^{\mathbb{E}_{\Theta}}=\left\{\left(U_{i}, \psi_{i}^{\mathbb{E}_{\Theta}}\right)\right\}_{i \in I}$ is an atlas of $\mathbb{E}_{\Theta}$.

Definition 3.1 Let $\mathrm{d}$ be a compatible distance in the flag manifold $\mathbb{F}_{\Theta}$. For a given $\varepsilon>0$ and an open covering $\mathcal{U}$ of $B$, a $\Psi$-adapted covering of $\mathbb{E}_{\Theta}$ is defined as

$$
\mathcal{U}_{\varepsilon}=\left\{\left(\psi_{i}^{\mathbb{E}_{\Theta}}\right)^{-1}\left(\left(U \cap U_{i}\right) \times \mathrm{B}_{\mathrm{d}}(u, \varepsilon)\right): U \in \mathcal{U}, i \in I, u \in \mathbb{F}_{\Theta}\right\} .
$$

The family of all $\Psi$-adapted coverings of $\mathbb{E}_{\Theta}$ is denoted by $\mathcal{O}_{\Theta}$.

The flag bundle can be reduced to a subbundle $P \rightarrow B$ whose structure group is $K$. Moreover, there is a compatible distance $\mathrm{d}$ in $\mathbb{F}_{\Theta}$ such that the elements of $K$ are isometries of $\left(\mathbb{F}_{\Theta}, \mathrm{d}\right)$. This implies that the family $\mathcal{O}_{\Theta}$ is admissible [16, Proposition 3.5], which essentially means that $\mathcal{O}_{\Theta}$ associates with a covering uniformity on $\mathbb{E}_{\Theta}[1]$.

For a given open covering $\mathcal{U}_{\varepsilon} \in \mathcal{O}_{\Theta}$, we define the $\operatorname{End}_{l}\left(\mathbb{E}_{\Theta}\right)$-neighborhood of the identity map relative to $\mathcal{U}_{\varepsilon}$ as

$\mathcal{N}_{\mathcal{U}_{\varepsilon}}=\left\{\phi \in \operatorname{End}_{l}\left(\mathbb{E}_{\Theta}\right)\right.$ : for every $x \in \operatorname{dom} \phi$, there is $U \in \mathcal{U}_{\varepsilon}$ such that $\left.x, \phi(x) \in U\right\}$.

The local semigroup of endomorphisms $\operatorname{End}_{l}\left(\mathbb{E}_{\Theta}\right)$ is $\mathcal{O}_{\Theta}$-locally transitive, that is, if $\mathcal{U}_{\varepsilon} \in \mathcal{O}_{\Theta}$ and $\left(\psi_{i}^{\mathbb{E}_{\Theta}}\right)^{-1}\left(\left(U \cap U_{i}\right) \times \mathrm{B}_{\mathrm{d}}(u, \varepsilon)\right) \in \mathcal{U}_{\varepsilon}$, for each pair of points $x, y \in$ $\left(\psi_{i}^{\mathbb{E}_{\Theta}}\right)^{-1}\left(\left(U \cap U_{i}\right) \times \mathrm{B}_{\mathrm{d}}(u, \varepsilon)\right)$, there is a $\phi \in \mathcal{N}_{\mathcal{U}_{\varepsilon}}$ such that $\phi(x)=y$ [16, Theorem 3.8].

Now, let $(S, Q, \sigma)$ be a left semigroup action on $Q$ with map $\sigma: S \times Q \rightarrow Q$ such that the transition $\sigma_{t}: Q \rightarrow Q$ is an automorphism of $Q$ for every $t \in S$. Consider the induced actions $\sigma^{B}$ and $\sigma^{\mathbb{E}_{\Theta}}$ of $S$, respectively, on $B$ and $\mathbb{E}_{\Theta}$. For establishing a direction for asymptotic behavior, we consider a filter basis $\mathcal{F}$ on the subsets of $S$ that satisfies the following hypotheses.

Definition $3.2[6,7]$ The filter basis $\mathcal{F}$ satisfies the Translation Hypothesis if the following conditions hold:

$\mathrm{H}_{1}$ for every $s \in S$ and $A \in \mathcal{F}$ there exists $B \in \mathcal{F}$ such that $s B \subset A$.

$\mathrm{H}_{2}$ for every $s \in S$ and $A \in \mathcal{F}$ there exists $B \in \mathcal{F}$ such that $B s \subset A$.

$\mathrm{H}_{3}$ for every $s \in S$ and $A \in \mathcal{F}$ there exists $B \in \mathcal{F}$ such that $B \subset A s$.

$\mathrm{H}_{4}$ for every $s \in S$ and $A \in \mathcal{F}$ there exists $B \in \mathcal{F}$ such that $B \subset s A$.

Properties $\mathrm{H}_{3}$ and $\mathrm{H}_{4}$ together mean that the semigroup $S$ is reversible, that is, $S s \cap S t \neq \emptyset$ and $s S \cap t S \neq \varnothing$ for all $s, t \in S$. By denoting $S^{-1}=\left\{t^{-1}: t \in S\right\}$, one has the reverse semigroup action $\left(S^{-1}, Q, \sigma^{*}\right)$ with map $\sigma_{t^{-1}}^{*}(q)=\sigma_{t}^{-1}(q)$ for all $t \in S$ and $q \in Q$. Then, $S$ satisfies the Ore conditions, that is, $T=S^{-1} S$ is the group generated by $S$ [9]. A set $X$ is invariant by $T$ if and only if it is invariant simultaneously by $S$ and $S^{-1}$.

Definition 3.3 A net $\left(t_{\lambda}\right)_{\lambda \in \Lambda}$ in $S$ diverges on the direction of $\mathcal{F}$ if for each $A \in \mathcal{F}$ there is $\lambda_{0} \in \Lambda$ such that $t_{\lambda} \in A$ for all $\lambda \geq \lambda_{0}$. The notation $t_{\lambda} \longrightarrow \mathcal{F} \infty$ means that $\left(t_{\lambda}\right) \mathcal{F}$-diverges. 
Definition 3.4 Let $\Theta \subset \Sigma$ and $X \subset \mathbb{E}_{\Theta}$. The $\omega$-limit set of $X$ with respect to $\mathcal{F}$ is given by $\omega(X, \mathcal{F})=\left\{\eta \in \mathbb{E}_{\Theta}:\right.$ there are nets $t_{\lambda} \longrightarrow \mathcal{F} \infty$ and $\left(\xi_{\lambda}\right)$ in $X$ such that $\left.t_{\lambda} \xi_{\lambda} \longrightarrow \eta\right\}$ and the $\omega^{*}$-limit set of $X$ with respect fo $\mathcal{F}$ by $\omega^{*}(\xi, \mathcal{F})=\left\{\eta \in \mathbb{E}_{\Theta}:\right.$ there are nets $t_{\lambda} \longrightarrow \mathcal{F} \infty$ and $\left(\xi_{\lambda}\right)$ in $X$ such that $\left.t_{\lambda}^{-1} \xi_{\lambda} \longrightarrow \eta\right\}$.

By Translation Hypothesis, $\omega$ and $\omega^{*}$-limit sets are invariant by $T$.

Definition 3.5 Let $\Theta \subset \Sigma$. For $\xi, \eta \in \mathbb{E}_{\Theta}, \mathcal{U}_{\varepsilon} \in \mathcal{O}_{\Theta}$, and $A \in \mathcal{F}$, we define an $\left(\mathcal{U}_{\varepsilon}, A\right)$ -chain from $\xi$ to $\eta$ as a finite sequence of points $x_{0}=\xi, x_{1}, \ldots, x_{n}=\eta$ in $\mathbb{E}_{\Theta}$, elements $t_{0}, \ldots, t_{n-1} \in A$, and open sets $U_{0}, \ldots, U_{n-1} \in \mathcal{U}_{\varepsilon}$ such that $t_{i} x_{i}, x_{i+1} \in U_{i}$, for $i=$ $0, \ldots, n-1$. The $\Omega$-chain limit set of $\xi \in \mathbb{E}_{\Theta}$ with respect to $\mathcal{F}$ is defined as

$$
\Omega(\xi, \mathcal{F})=\bigcap_{\mathcal{U}_{\varepsilon} \in \mathcal{O}_{\Theta}, A \in \mathcal{F}} \Omega\left(\xi, \mathcal{U}_{\varepsilon}, A\right),
$$

where $\Omega\left(\xi, \mathcal{U}_{\varepsilon}, A\right)=\left\{\eta \in \mathbb{E}_{\Theta}\right.$ : there is an $\left(\mathcal{U}_{\varepsilon}, A\right)$-chain from $\xi$ to $\left.\eta\right\}$; the $\Omega^{*}$-chain limit set of $\xi$ with respect to $\mathcal{F}$ is defined as

$$
\Omega^{*}(\xi, \mathcal{F})=\bigcap_{\mathcal{U}_{\varepsilon} \in \mathcal{O}_{\Theta}, A \in \mathcal{F}} \Omega^{*}\left(\xi, \mathcal{U}_{\varepsilon}, A\right),
$$

where $\Omega^{*}\left(\xi, \mathcal{U}_{\varepsilon}, A\right)=\left\{\eta \in \mathbb{E}_{\Theta}\right.$ : there is an $\left(\mathcal{U}_{\varepsilon}, A\right)$-chain from $\xi$ to $\left.\eta\right\}$. A point $\xi \in \mathbb{E}_{\Theta}$ is $\mathcal{F}$-chain recurrent if $\xi \in \Omega(\xi, \mathcal{F})$. A subset $X \subset \mathbb{E}_{\Theta}$ is $\mathcal{F}$-chain recurrent if all points in $X$ are $\mathcal{F}$-chain recurrent. A subset $X \subset \mathbb{E}_{\Theta}$ is $\mathcal{F}$-chain transitive if $X \subset \Omega(\xi, \mathcal{F})$ for all $\xi \in X$. We denote by $\mathfrak{R}_{\Theta}$ the $\mathcal{F}$-chain recurrence set, that is, the set of all $\mathcal{F}$-chain recurrent points of $S$ in $\mathbb{E}_{\Theta}$.

The maximal $\mathcal{F}$-chain transitive sets of $S$ in $\mathbb{E}_{\Theta}$ are given by

$$
E_{\xi}=\Omega(\xi, \mathcal{F}) \cap \Omega^{*}(\xi, \mathcal{F})
$$

with $\xi \in \mathfrak{R}_{\Theta}$.

Remark 3.1 The $\mathcal{F}$-chain recurrence set $\mathfrak{R}_{\Theta}$ and the maximal $\mathcal{F}$-chain transitive sets are closed [6, Propositions 4.6 and 4.7].

Remark 3.2 For a given $\xi \in \mathbb{E}_{\Theta}$, we have $\Omega(\xi, \mathcal{F})=\bigcap_{\mathcal{U}_{\varepsilon} \in \mathcal{O}_{\Theta}, A \in \mathcal{F}} \omega\left(\Omega\left(\xi, \mathcal{U}_{\varepsilon}, A\right)\right)[6$, Proposition 4.9]. Hence, the $\Omega$-chain limit sets are invariant by $T$. For $\eta \in \Omega^{*}(\xi, \mathcal{F}), s \in S$, $\mathcal{U}_{\varepsilon} \in \mathcal{O}_{\Theta}$, and $A \in \mathcal{F}$, take $B \in \mathcal{F}$ such that $B \subset A \cap A s \cap A s^{-1}$. There are sequences $x_{0}=$ $\eta, x_{1}, \ldots, x_{n}=\xi$ in $\mathbb{E}_{\Theta}, t_{0}, \ldots, t_{n-1} \in B$, and $U_{0}, \ldots, U_{n-1} \in \mathcal{U}_{\varepsilon}$ such that $t_{i} x_{i}, x_{i+1} \in U_{i}$. Then, $t_{0}=t_{0}^{\prime} s=t_{0}^{\prime \prime} s^{-1}$, for some $t_{0}^{\prime}, t_{0}^{\prime \prime} \in A$, and hence $t_{0}^{\prime} s \eta=t_{0}^{\prime \prime} s^{-1} \eta=t_{0} \eta \in U_{0}$. This means that $s \eta, s^{-1} \eta \in \Omega^{*}\left(\xi, \mathcal{U}_{\varepsilon}, A\right)$. Hence, the $\Omega^{*}$-chain limit sets are also invariant by $T$, and therefore the maximal $\mathcal{F}$-chain transitive sets are invariant by $T$.

Remark 3.3 A compact, connected, and $\mathcal{F}$-chain recurrent set is $\mathcal{F}$-chain transitive [7, Proposition 4.5].

Remark 3.4 For every $\xi \in \mathbb{E}_{\Theta}$, we have $\omega(\xi, \mathcal{F}) \subset \Omega(\xi, \mathcal{F})$ and $\omega^{*}(\xi, \mathcal{F}) \subset \Omega^{*}(\xi, \mathcal{F})$. Hypothesis $\mathrm{H}_{3}$ implies the limit set $\omega(\xi, \mathcal{F})$ is $\mathcal{F}$-chain transitive if it is nonempty [6, Proposition 4.5]. By using the hypothesis $\mathrm{H}_{4}$, we can see that the limit set $\omega^{*}(\xi, \mathcal{F})$ is also $\mathcal{F}$-chain transitive. Hence, $\eta \in \omega(\xi, \mathcal{F})$ (or $\eta \in \omega^{*}(\xi, \mathcal{F})$ ) implies $\eta \in \mathfrak{R}_{\Theta}$ and $\omega(\xi, \mathcal{F}) \subset E_{\eta}$ (or $\left.\omega^{*}(\xi, \mathcal{F}) \subset E_{\eta}\right)$. 
Definition 3.6 The domain of $\mathcal{F}$-chain attraction and the domain of $\mathcal{F}$-chain repellency of a maximal $\mathcal{F}$-chain transitive set $E$ are defined, respectively, by

$$
\begin{aligned}
\mathcal{A}(E) & =\left\{\eta \in \mathbb{E}_{\Theta}: \Omega(\eta, \mathcal{F}) \cap E \neq \emptyset\right\}, \\
\mathcal{A}^{*}(E) & =\left\{\eta \in \mathbb{E}_{\Theta}: \Omega^{*}(\eta, \mathcal{F}) \cap E \neq \emptyset\right\} .
\end{aligned}
$$

Note that $E=\mathcal{A}(E) \cap \mathcal{A}^{*}(E)$. The dynamic ordering among the maximal $\mathcal{F}$-chain transitive sets is given as the following: For two maximal $\mathcal{F}$-chain transitive sets $E$ and $E^{\prime}$, $E \preceq E^{\prime}$ means $E \subset \mathcal{A}\left(E^{\prime}\right)$.

A description of the maximal chain transitive sets is provided in terms of the action of shadowing semigroups. The shadowing semigroups on $\mathbb{E}_{\Theta}$ are perturbations of $S$ in the local semigroup $\operatorname{End}_{l}\left(\mathbb{E}_{\Theta}\right)$, as follows.

Definition 3.7 For each $\mathcal{U}_{\varepsilon} \in \mathcal{O}_{\Theta}$ and $A \in \mathcal{F}$, define the set

$$
\mathcal{N}_{\mathcal{U}_{\varepsilon}, A}=\left\{\phi s: \phi \in \mathcal{N}_{\mathcal{U}_{\varepsilon}}, s \in A\right\} .
$$

The $\left(\mathcal{U}_{\varepsilon}, A\right)$-shadowing semigroup $\operatorname{End}_{l}\left(\mathbb{E}_{\Theta}\right)_{\mathcal{U}_{\varepsilon}, A}$ is the local semigroup generated by $\mathcal{N}_{\mathcal{U}_{\varepsilon}, A}$.

For every $\xi \in \mathbb{E}_{\Theta}, \mathcal{U}_{\varepsilon} \in \mathcal{O}_{\Theta}$, and $A \in \mathcal{F}$, we have

$$
\operatorname{End}_{l}\left(\mathbb{E}_{\Theta}\right)_{\mathcal{U}_{\varepsilon}, A} \xi=\Omega\left(\xi, \mathcal{U}_{\varepsilon}, A\right) \quad \text { and } \operatorname{End}_{l}\left(\mathbb{E}_{\Theta}\right)_{\mathcal{U}_{\varepsilon}, A}^{*} \xi=\Omega^{*}\left(\xi, \mathcal{U}_{\varepsilon}, A\right)
$$

[24, Proposition 3.3]. We denote by $\mathcal{S}_{\mathcal{U}_{\varepsilon}, A} \subset \operatorname{End}_{l}(Q)$ the local subsemigroup of $\operatorname{End}_{l}(Q)$ such that $\left(\mathcal{S}_{\mathcal{U}_{\varepsilon}, A}\right)_{\mathbb{E}_{\Theta}}=\operatorname{End}_{l}\left(\mathbb{E}_{\Theta}\right)_{\mathcal{U}_{\varepsilon}, A}$. The action of $S$ on the base space $B$ is $\mathcal{F}$-chain transitive if and only if each shadowing semigroup $\left(\mathcal{S}_{\mathcal{U}_{\varepsilon}, A}\right)_{B}$ is transitive [24, Proposition 4.4]. Thus, $B$ is a control set for each semigroup $\left(\mathcal{S}_{\mathcal{U}_{\varepsilon}, A}\right)_{B}$ if the action of $S$ on the base space $B$ is $\mathcal{F}$-chain transitive. In this case, the control sets of $\left(\mathcal{S}_{\mathcal{U}_{\varepsilon}, A}\right)_{\mathbb{E}_{\Theta}}=\operatorname{End}_{l}\left(\mathbb{E}_{\Theta}\right)_{\mathcal{U}_{\varepsilon}, A}$ in $\mathbb{E}_{\Theta}$ are $\mathbb{D}_{\mathcal{U}_{\varepsilon}, A}^{\Theta}(w)$ with $w \in W$, where $\mathbb{D}_{\mathcal{U}_{\varepsilon}, A}^{\Theta}(w)_{0} \cap\left(\mathbb{E}_{\Theta}\right)_{x} \neq \emptyset$, for all $x \in B$.

The following theorem is proved in [24, Theorems 3.1 and 4.1].

Theorem 3.1 Assume that the base space B is $\mathcal{F}$-chain transitive. Let $\Theta$ be a set in the root simple system $\Sigma$. For each $w$ in the Weyl group $W$, set

$$
E_{\Theta}(w)=\bigcap_{\mathcal{U}_{\varepsilon} \in \mathcal{O}_{\Theta}, A \in \mathcal{F}} \operatorname{cls}\left(\mathbb{D}_{\mathcal{U}_{\varepsilon}, A}^{\Theta}(w)_{0}\right)
$$

in the flag bundle $\mathbb{E}_{\Theta}$, where $\mathbb{D}_{\mathcal{U}_{\varepsilon}, A}(w)$ is the $w$-control set of the shadowing semigroup End $_{l}\left(\mathbb{E}_{\Theta}\right)_{\mathcal{U}_{\varepsilon}, A}$. Then, $\left\{E_{\Theta}(w): w \in W\right\}$ is the collection of all maximal $\mathcal{F}$-chain transitive sets of $S$ in $\mathbb{E}_{\Theta}$ and

$$
E_{\Theta}(w)=\bigcap_{\mathcal{U}_{\varepsilon}, A} \mathbb{D}_{\mathcal{U}_{\varepsilon}, A}^{\Theta}(w)_{0}=\bigcap_{\mathcal{U}_{\varepsilon}, A} \mathbb{D}_{\mathcal{U}_{\varepsilon}, A}^{\Theta}(w) .
$$

Since the Weyl group $W$ is a finite group, the number of maximal $\mathcal{F}$-chain transitive sets of $S$ on $\mathbb{E}_{\Theta}$ is finite. The following theorem relates the dynamic ordering among the maximal $\mathcal{F}$-chain transitive sets to the dynamic ordering among the control sets of the shadowing semigroups.

Theorem 3.2 Assume that the base space B is $\mathcal{F}$-chain transitive. Let $\Theta$ be a set in the root simple system $\Sigma$. For each $w$ in the Weyl group $W$, one has

$$
\begin{gathered}
\mathcal{A}\left(E_{\Theta}(w)\right)=\bigcap_{\mathcal{U}_{\varepsilon} \in \mathcal{O}_{\Theta}, A \in \mathcal{F}} \mathcal{A}\left(\mathbb{D}_{\mathcal{U}_{\varepsilon}, A}^{\Theta}(w)\right), \\
\mathcal{A}_{\mathcal{O}}^{*}\left(E_{\Theta}(w)\right)=\bigcap_{\mathcal{U}_{\varepsilon} \in \mathcal{O}_{\Theta}, A \in \mathcal{F}} \mathcal{A}^{*}\left(\mathbb{D}_{\mathcal{U}_{\varepsilon}, A}^{\Theta}(w)\right) .
\end{gathered}
$$


In particular, if $\mathbb{D}_{\mathcal{U}_{\varepsilon}, A}^{\Theta}(w)$ is an invariant control set of $\operatorname{End}_{l}\left(\mathbb{E}_{\Theta}\right)_{\mathcal{U}_{\varepsilon}, A}$, for all $\mathcal{U}_{\varepsilon} \in \mathcal{O}_{\Theta}$ and $A \in \mathcal{F}$, then $E_{\Theta}(w)$ is maximal with respect to the dynamic ordering among the maximal $\mathcal{F}$-chain transitive sets.

Proof For a given $\xi \in \mathcal{A}\left(E_{\Theta}(w)\right)$, take $\eta \in \Omega(\xi, \mathcal{F}) \cap E_{\Theta}(w)$. By Theorem 3.1, we have $E_{\Theta}(w)=\bigcap_{\mathcal{U}_{\varepsilon}, A} \mathbb{D}_{\mathcal{U}_{\varepsilon}, A}^{\Theta}(w)_{0}$, hence $\eta \in \operatorname{End}_{l}\left(\mathbb{E}_{\Theta}\right)_{\mathcal{U}_{\varepsilon}, A} \xi \cap \mathbb{D}_{\mathcal{U}_{\varepsilon}, A}^{\Theta}(w)_{0}$ for all $\mathcal{U}_{\varepsilon} \in \mathcal{O}_{\Theta}$ and $A \in \mathcal{F}$. Therefore, $\xi \in \mathcal{A}\left(\mathbb{D}_{\mathcal{U}_{\varepsilon}, A}^{\Theta}(w)\right)$ for all $\mathcal{U}_{\varepsilon} \in \mathcal{O}_{\Theta}$ and $A \in \mathcal{F}$. As to the converse, if $\xi \in \bigcap_{\mathcal{U}_{\varepsilon}, A} \mathcal{A}\left(\mathbb{D}_{\mathcal{U}_{\varepsilon}, A}^{\Theta}(w)\right)$, then there is $\xi_{\mathcal{U}_{\varepsilon}, A} \in \operatorname{End}_{l}\left(\mathbb{E}_{\Theta}\right)_{\mathcal{U}_{\varepsilon}, A} \xi \cap \mathbb{D}_{\mathcal{U}_{\varepsilon}, A}^{\Theta}(w)_{0}$, for all $\mathcal{U}_{\varepsilon} \in \mathcal{O}_{\Theta}$ and $A \in \mathcal{F}$. For a given $\eta \in E_{\Theta}(w) \subset \mathbb{D}_{\mathcal{U}_{\varepsilon}, A}^{\Theta}(w)_{0}$, we have $\eta \in \operatorname{End}_{l}\left(\mathbb{E}_{\Theta}\right)_{\mathcal{U}_{\varepsilon}, A} \xi_{\mathcal{U}_{\varepsilon}, A} \subset \operatorname{End}_{l}\left(\mathbb{E}_{\Theta}\right)_{\mathcal{U}_{\varepsilon}, A} \xi$ for all $\mathcal{U}_{\varepsilon} \in \mathcal{O}_{\Theta}$ and $A \in \mathcal{F}$. This means that $\eta \in \Omega(\xi, \mathcal{F}) \cap E_{\Theta}(w)$, and thus $\xi \in \mathcal{A}\left(E_{\Theta}(w)\right)$. Therefore, $\mathcal{A}\left(E_{\Theta}(w)\right)=$ $\bigcap_{\mathcal{U}_{\varepsilon}, A} \mathcal{A}\left(\mathbb{D}_{\mathcal{U}_{\varepsilon}, A}^{\Theta}(w)\right)$. The other equality follows by similar way. Now, suppose that $\mathbb{D}_{\mathcal{U}_{\varepsilon}, A}^{\Theta}(w)$ is an invariant control set of $\operatorname{End}_{l}\left(\mathbb{E}_{\Theta}\right)_{\mathcal{U}_{\varepsilon}, A}$, for all $\mathcal{U}_{\varepsilon} \in \mathcal{O}_{\Theta}$ and $A \in \mathcal{F}$. If $E_{\Theta}(w) \preceq E_{\Theta}\left(w^{\prime}\right)$, then $E_{\Theta}(w) \subset \mathcal{A}\left(E_{\Theta}\left(w^{\prime}\right)\right)=\bigcap_{\mathcal{U}_{\varepsilon}, A} \mathcal{A}\left(\mathbb{D}_{\mathcal{U}_{\varepsilon}, A}\left(w^{\prime}\right)\right)$. This means that $\mathbb{D}_{\mathcal{U}_{\varepsilon}, A}^{\Theta}(w) \cap \mathcal{A}\left(\mathbb{D}_{\mathcal{U}_{\varepsilon}, A}^{\Theta}\left(w^{\prime}\right)\right) \neq \emptyset$, that is, $\mathbb{D}_{\mathcal{U}_{\varepsilon}, A}^{\Theta}(w) \preceq \mathbb{D}_{\mathcal{U}_{\varepsilon}, A}^{\Theta}\left(w^{\prime}\right)$ for all $\mathcal{U}_{\varepsilon} \in \mathcal{O}_{\Theta}$ and $A \in \mathcal{F}$. Since $\mathbb{D}_{\mathcal{U}_{\varepsilon}, A}^{\Theta}(w)$ is maximal with respect to the dynamic ordering among the control sets of $\operatorname{End}_{l}\left(\mathbb{E}_{\Theta}\right)_{\mathcal{U}_{\varepsilon}, A}$, it follows that $\mathbb{D}_{\mathcal{U}_{\varepsilon}, A}^{\Theta}(w)=\mathbb{D}_{\mathcal{U}_{\varepsilon}, A}^{\Theta}\left(w^{\prime}\right)$, for all $\mathcal{U}_{\varepsilon} \in \mathcal{O}_{\Theta}$ and $A \in \mathcal{F}$, and hence $E_{\Theta}(w)=E_{\Theta}\left(w^{\prime}\right)$. Therefore, $E_{\Theta}(w)$ is maximal with respect to the dynamic ordering among the maximal $\mathcal{F}$-chain transitive sets.

The dynamic ordering among the maximal $\mathcal{F}$-chain transitive sets relates to the BruhatChevalley order on the Weyl group $W[18$, Section 4]. To see this relation, we need the concept of parabolic type of semigroups. Let $R \subset G$ be a semigroup with interior points. For a given $\Theta \subset \Sigma$, let $\mathbb{A}_{\Theta}$ (1) be the invariant control set of $R$ in the flag manifold $\mathbb{F}_{\Theta}$. According to [21], there exists a unique set $\Theta(R) \subset \Sigma$ that is maximal with the property that $\pi_{\Theta}^{-1}\left(\mathbb{A}_{\Theta}(1)\right)=\mathbb{A}(1)$, where $\mathbb{A}(1)$ is the invariant control set of $R$ in the maximal flag manifold $\mathbb{F}$. The subset $\Theta(R)$ is called parabolic type of $R$. If $R_{1} \subset R_{2}$ are semigroups of $G$ and $\mathbb{A}_{\Theta}^{1}(1), \mathbb{A}_{\Theta}^{2}(1)$ are, respectively, their invariant control sets in $\mathbb{F}_{\Theta}$, one has $\mathbb{A}_{\Theta}^{1}(1) \subset \mathbb{A}_{\Theta}^{2}(1)$, and therefore $\Theta\left(R_{1}\right) \subset \Theta\left(R_{2}\right)$.

For a local semigroup of endomorphisms $\mathcal{S} \subset \operatorname{End}_{l}(Q)$, the parabolic type $\Theta\left(\mathcal{S}_{q}\right)$ of $\mathcal{S}_{q}$ does not depend on the point $q \in Q$ [16, Proposition 5.10]. Then, $\Theta(\mathcal{S})=\Theta\left(\mathcal{S}_{q}\right)$ (for any $q$ ) defines the parabolic type of $\mathcal{S}$. Let $\mathbb{D}(1)$ be the invariant control set of $\mathcal{S}$ such that $\mathbb{D}(1) \cap(\mathbb{E})_{\pi(q)}=q \cdot \mathbb{A}(1)$. Then, the subgroup $W(\mathcal{S})=\{w \in W: \mathbb{D}(w)=\mathbb{D}(1)\}$ of $W$ is parabolic of type $\Theta(\mathcal{S})$, that is, $W(\mathcal{S})=W_{\Theta(\mathcal{S})}$ is the subgroup of $W$ generated by the set of reflections $\left\{r_{\alpha}: \alpha \in \Theta(\mathcal{S})\right\}$.

The following result relates the dynamic ordering among the control sets of a local semigroup to the Bruhat-Chevalley order on the Weyl group $W$. A proof for it can be found in [15, Theorem 4.36].

Proposition 3.1 Let $\mathcal{S} \subset \operatorname{End}_{l}(Q)$ be a local semigroup whose forward and backward orbits in $Q$ are open sets. One has $\mathbb{D}(w) \preceq \mathbb{D}\left(w^{\prime}\right)$ if and only if $W(\mathcal{S}) w \geqslant W(\mathcal{S}) w^{\prime}$, where " $\geqslant$ " means the Bruhat-Chevalley order on the quotient $W(\mathcal{S}) \backslash W$. In particular, $\mathbb{D}^{\Theta}(w)=$ $\mathbb{D}^{\Theta}\left(w^{\prime}\right)$ if and only if $W(\mathcal{S}) w W_{\Theta}=W(\mathcal{S}) w^{\prime} W_{\Theta}$. Furthermore, the control set $\mathbb{D}^{\Theta}(1)$ is the unique maximal and $\mathbb{D}^{\Theta}\left(w_{0}\right)$ is the unique minimal, where $w_{0}$ is the principal involution of $W$ with respect to $\Sigma$. 

$\mathbb{E}$.

For the following, we denote $\mathcal{O}=\mathcal{O}_{\emptyset}$ the admissible family on the maximal flag bundle

Definition 3.8 The parabolic type of the semigroup action $(S, Q, \sigma)$ is given by

$$
\Theta(\sigma)=\bigcap_{\mathcal{U}_{\varepsilon} \in \mathcal{O}, A \in \mathcal{F}} \Theta\left(\mathcal{S}_{\mathcal{U}_{\varepsilon}, A}\right)
$$

and $W(\sigma)$ denotes the parabolic subgroup of $W$ of type $\Theta(\sigma)$.

Lemma 3.1 There exist $\mathcal{U}_{\varepsilon_{0}}^{0} \in \mathcal{O}$ and $A_{0} \in \mathcal{F}$ such that $\Theta(\sigma)=\Theta\left(\mathcal{S}_{\mathcal{U}_{\varepsilon_{0}}^{0} A_{0}}\right)$.

Proof Since the simple root system $\Sigma$ is finite, the number of possible parabolic types is finite. Suppose that $\Theta\left(\mathcal{S}_{\mathcal{U}_{\varepsilon_{k}}^{k}, A_{k}}\right), k=1, \ldots, n$, are all the parabolic types of the shadowing semigroups. Take $\mathcal{U}_{\varepsilon_{0}}^{0} \in \mathcal{O}$ and $A_{0} \in \mathcal{F}$ such that $\mathcal{U}_{\varepsilon_{0}}^{0}$ refines $\mathcal{U}_{\varepsilon_{k}}^{k}$, for all $k$, and $A_{0} \subset \bigcap_{k=1}^{n} A_{k}$. We have $\operatorname{End}_{l}\left(\mathbb{E}_{\Theta}\right)_{\mathcal{U}_{\varepsilon_{0}}^{0}, A_{0}} \subset \bigcap_{k=1}^{n} \operatorname{End}_{l}\left(\mathbb{E}_{\Theta}\right)_{\mathcal{U}_{\varepsilon_{k}}^{k}, A_{k}}$, and therefore

$$
\Theta\left(\mathcal{S}_{\mathcal{U}_{\varepsilon_{0}}^{0}, A_{0}}\right) \subset \bigcap_{k=1}^{n} \Theta\left(\mathcal{S}_{\mathcal{U}_{\varepsilon_{k}}^{k}, A_{k}}\right)=\Theta(\sigma) \subset \Theta\left(\mathcal{S}_{\mathcal{U}_{\varepsilon_{0}}^{0}, A_{0}}\right) .
$$

The reverse parabolic type of $(S, Q, \sigma)$ is the dual

$$
\Theta^{*}(\sigma)=-w_{0} \Theta(\sigma)=\bigcap_{\mathcal{U}_{\varepsilon} \in \mathcal{O}, A \in \mathcal{F}}-w_{0} \Theta\left(\mathcal{S}_{\mathcal{U}_{\varepsilon}, A}\right)
$$

(cf. [19]). By Lemma 3.1 and [19, Proposition 6.2], we have $\Theta^{*}(\sigma)=\Theta\left(\left(\mathcal{S}_{\mathcal{U}_{\varepsilon_{0}}^{0}, A_{0}}\right)_{q}^{-1}\right)$ (for any $q \in Q$ ).

We now relate the dynamic ordering among the maximal chain transitive sets to the Bruhat-Chevalley order on $W$.

Theorem 3.3 One has $E(w) \preceq E\left(w^{\prime}\right)$ if and only if $W(\sigma) w \geqslant W(\sigma) w^{\prime}$. In particular, $E_{\Theta}(w)=E_{\Theta}\left(w^{\prime}\right)$ if and only if $W(\sigma) w W_{\Theta}=W(\sigma) w^{\prime} W_{\Theta}$. Furthermore, $E_{\Theta}$ (1) is the only maximal and $E_{\Theta}\left(w_{0}\right)$ is the only minimal.

Proof Take $\mathcal{U}_{\varepsilon_{0}} \in \mathcal{O}$ and $A_{0} \in \mathcal{F}$ such that $\Theta(\sigma)=\Theta\left(\mathcal{S}_{\mathcal{U}_{\varepsilon_{0}}, A_{0}}\right)$, as in Lemma 3.1. Then, $W(\sigma)=W\left(\mathcal{S}_{\mathcal{U}_{\varepsilon_{0}}, A_{0}}\right)$ and $E(w) \subset \mathbb{D}_{\mathcal{U}_{\varepsilon_{0}}, A_{0}}(w)_{0}$ for every $w \in W$. If $E(w) \preceq$ $E\left(w^{\prime}\right)$, then there is $\xi \in E(w)$ and $\xi^{\prime} \in E\left(w^{\prime}\right)$ such that $\xi^{\prime} \in \Omega(\xi, \mathcal{F})$. Hence, $\xi^{\prime} \in$ $\operatorname{End}_{l}(\mathbb{E})_{\mathcal{U}_{\varepsilon_{0}}, A_{0}} \xi$, and therefore $\mathbb{D}_{\mathcal{U}_{\varepsilon_{0}}, A_{0}}(w) \preceq \mathbb{D}_{\mathcal{U}_{\varepsilon_{0}}, A_{0}}\left(w^{\prime}\right)$. By Proposition 3.1, it follows that $W(\sigma) w \geqslant W(\sigma) w^{\prime}$. On the other hand, let $w, w^{\prime} \in W$ with $W(\sigma) w \geqslant W(\sigma) w^{\prime}$. For $\mathcal{V}_{\varepsilon} \in \mathcal{O}$ and $A \in \mathcal{F}$, take $\mathcal{W}_{\delta} \in \mathcal{O}$ and $B \in \mathcal{F}$ such that $\mathcal{W}_{\delta}$ refines both $\mathcal{U}_{\varepsilon_{0}}, \mathcal{V}_{\varepsilon}$ and $B \subset A \cap A_{0}$. Then, $\mathcal{S}_{\mathcal{W}_{\delta}, B} \subset \mathcal{S}_{\mathcal{U}_{\varepsilon_{0}}, A_{0}}$, and hence $\Theta(\sigma)=\Theta\left(\mathcal{S}_{\mathcal{W}_{\delta}, B}\right)$. Moreover, by Proposition 3.1, we have $\mathbb{D}_{\mathcal{W}_{\delta}, B}(w) \preceq \mathbb{D}_{\mathcal{W}_{\delta}, B}\left(w^{\prime}\right)$. Since $E(w) \subset \mathbb{D}_{\mathcal{W}_{\delta}, B}(w)_{0}$ and $E\left(w^{\prime}\right) \subset \mathbb{D}_{\mathcal{W}_{\delta}, B}\left(w^{\prime}\right)_{0}$, it follows that $\xi^{\prime} \in \operatorname{End}_{l}(\mathbb{E})_{\mathcal{W}_{\delta}, B} \xi$, for all $\xi \in E(w)$ and $\xi^{\prime} \in$ $E\left(w^{\prime}\right)$. Hence, $\xi^{\prime} \in \Omega\left(\xi, \mathcal{W}_{\delta}, B\right) \subset \Omega\left(\xi, \mathcal{V}_{\varepsilon}, A\right)$, for all $\xi \in E(w)$ and $\xi^{\prime} \in E\left(w^{\prime}\right)$. This means that $E(w) \preceq E\left(w^{\prime}\right)$.

From now on, we denote $\Theta_{\mathcal{U}_{\varepsilon}, A}=\Theta\left(\mathcal{S}_{\mathcal{U}_{\varepsilon}, A}\right)$ for all $\mathcal{U}_{\varepsilon} \in \mathcal{O}$ and $A \in \mathcal{F}$. The property of $\Theta_{\mathcal{U}_{\varepsilon}, A}$ transfers to $\Theta(\sigma)$, as the following. 
Proposition 3.2 The maximal $\mathcal{F}$-chain transitive sets $E_{\Theta(\sigma)}(1)$ and $E_{\Theta^{*}(\sigma)}\left(w_{0}\right)$ in the flag bundles $\mathbb{E}_{\Theta(\sigma)}$ and $\mathbb{E}_{\Theta *(\sigma)}$ satisfy

$$
\tilde{\pi}_{\Theta(\sigma)}^{-1}\left(E_{\Theta(\sigma)}(1)\right)=E(1) \quad \text { and } \quad \tilde{\pi}_{\Theta^{*}(\sigma)}^{-1}\left(E_{\Theta^{*}(\sigma)}\left(w_{0}\right)\right)=E\left(w_{0}\right) .
$$

Proof Take $\mathcal{U}_{\varepsilon_{0}} \in \mathcal{O}$ and $A_{0} \in \mathcal{F}$ such that $\Theta(\sigma)=\Theta_{\mathcal{U}_{\varepsilon_{0}}, A_{0}}$. For $\mathcal{V}_{\varepsilon} \in \mathcal{O}$ and $A \in \mathcal{F}$, take $\mathcal{W}_{\delta} \in \mathcal{O}$ and $B \in \mathcal{F}$ such that $\mathcal{W}_{\delta}$ refines both $\mathcal{U}_{\varepsilon_{0}}, \mathcal{V}_{\varepsilon}$ and $B \subset A \cap A_{0}$. We have $\mathbb{D}_{\mathcal{W}_{\delta}, B}(1)_{0} \subset \mathbb{D}_{\mathcal{V}_{\varepsilon}, A}(1)_{0}$ and $\Theta_{\mathcal{W}_{\delta}, B}=\Theta(\sigma)$. Now, if $q \cdot u \in \tilde{\pi}_{\Theta(\sigma)}^{-1}\left(E_{\Theta(\sigma)}(1)\right)$, then

$$
q \cdot u \in q \cdot \pi_{\Theta(\sigma)}^{-1}\left(\mathbb{A}_{\mathcal{W}_{\delta}, B}^{\Theta(\sigma)}(1)_{0}\right)=q \cdot \mathbb{A}_{\mathcal{W}_{\delta}, B}(1)_{0} \subset \mathbb{D}_{\mathcal{V}_{\varepsilon}, A}(1)_{0} .
$$

Hence, $q \cdot u \in E(1)$, and thus $\tilde{\pi}_{\Theta(\sigma)}^{-1}\left(E_{\Theta(\sigma)}(1)\right) \subset E$ (1). Since the inclusion $E$ (1) $\subset$ $\tilde{\pi}_{\Theta(\sigma)}^{-1}\left(E_{\Theta(\sigma)}(1)\right)$ holds in general, we have $\tilde{\pi}_{\Theta(\sigma)}^{-1}\left(E_{\Theta(\sigma)}(1)\right)=E(1)$. Similarly, by using the equality $\mathbb{A}_{\mathcal{U}_{\varepsilon}, A}^{\Theta^{*}(\sigma)}\left(w_{0}\right)_{0}=\mathbb{A}_{\mathcal{U}_{\varepsilon}, A}^{\Theta^{*}(\sigma)}(1)_{0}^{-}$, where $\mathbb{A}_{\mathcal{U}_{\varepsilon}, A}^{\Theta *}(1)^{-}$is the invariant control set of $\left(\mathcal{S}_{\mathcal{U}_{\varepsilon}, A}\right)_{q}^{-1}$, we have $\tilde{\pi}_{\Theta^{*}(\sigma)}^{-1}\left(E_{\Theta^{*}(\sigma)}\left(w_{0}\right)\right)=E\left(w_{0}\right)$.

This property of $E_{\Theta(\sigma)}(1)$ and $E_{\Theta^{*}(\sigma)}\left(w_{0}\right)$ will be used afterward in the main theorem.

\section{Algebraic description of maximal chain transitive sets}

This section contains the main results of the paper. We show that the flag bundle of parabolic type admits an equivariant global cross section. This is used to determine the one-parameter subgroup whose fixed points describe the intersection of the maximal chain transitive sets with the fibers of a flag bundle. Throughout, there is a connected semi-simple Lie group $G$ with Lie algebra $\mathfrak{g}$, an admissible triple $\left(\theta, \mathfrak{a}, \mathfrak{a}^{+}\right)$, a locally trivial principal bundle $\pi: Q \rightarrow B$ with structure group $G$, a semigroup of automorphisms $(S, Q, \sigma)$, and a filter basis $\mathcal{F}$ on the subsets of $S$ satisfying the Translation Hypothesis. Let $T=S^{-1} S$ be the group generated by $S$.

Denote $A^{+}=\exp \left(\mathfrak{a}^{+}\right)$and consider a polar decomposition $G=K \operatorname{cls}\left(A^{+}\right) K$, where $K$ is the compact subgroup of the Iwasawa decomposition of $G$ [12, Chapter IX]. Since the exponential map is bijective onto the connected subgroup $A=\langle\exp (\mathfrak{a})\rangle$, we can define the function $\lambda_{\alpha}(h)=\mathrm{e}^{\alpha(\log (h))}$, for every root $\alpha \in \Pi$ and $h \in A$. For a given subset $\Theta \subset \Sigma$, let $\mathfrak{n}_{\Theta}^{-}$be the nilpotent radical

$$
\mathfrak{n}_{\Theta}^{-}=\sum_{\alpha \in \Pi^{+}-\langle\Theta\rangle^{+}} \mathfrak{g}_{-\alpha}
$$

of the subalgebra $\theta\left(\mathfrak{p}_{\Theta}\right)$, and denote $N_{\Theta}^{-}=\exp \left(\mathfrak{n}_{\Theta}^{-}\right)$the connected subgroup with Lie algebra $\mathfrak{n}_{\Theta}^{-}$. For a given $h \in A$, the set of fixed point of $h$ is given by $\left\{w b_{\Theta}^{+}: w \in W\right\}$. For each $w \in W$, the Bruhat cell $N_{\Theta}^{-} w b_{\Theta}^{+}$is the stable manifold of $w b_{\Theta}^{+}$in the flag manifold $\mathbb{F}_{\Theta}$. The cell $N_{\Theta}^{-} b_{\Theta}^{+}$is the only one that is open, and for each $g \in G, g N_{\Theta}^{-} b_{\Theta}^{+}$is called an open Bruhat cell [20, Section 1]. Any open Bruhat cell is diffeomorphic with an Euclidean space. Moreover, $N_{\Theta}^{-} b_{\Theta}^{+}$is dense in $\mathbb{F}_{\Theta}$ and $b_{\Theta}^{+}$is the only attractor of $h \in A$, that is, $h^{n} x \rightarrow b_{\Theta}^{+}$ as $n \rightarrow \infty$, for every $x \in N_{\Theta}^{-} b_{\Theta}^{+}$.

The concept of contractible sequences in $G$ presented in [3,22] can be interpreted as contractible nets in $G$. For a given net $\left(g_{\iota}\right)_{\iota \in \mathcal{I}}$ in $G$, we write $g_{\iota}=u_{\iota} h_{\iota} v_{\iota}$, with $u_{\iota}, v_{\iota} \in K$ and $h_{\iota} \in \operatorname{cls}\left(A^{+}\right)$. We say that $\left(g_{\iota}\right)$ is admissible if $u_{\iota} \rightarrow u, v_{\iota} \rightarrow v, u, v \in K$, and the net $\lambda_{\alpha}\left(h_{\iota}\right)$ is convergent for every negative roots $\alpha$. Since $\lambda_{\alpha}\left(h_{\iota}\right) \in(0,1]$, any net has an admissible subnet. The numbers $\lambda_{\alpha}\left(h_{l}\right)$ together with 1 are the eigenvalues of $\operatorname{Ad}\left(h_{l}\right)$. Hence, 
the net of restrictions $\left(\left.\operatorname{Ad}\left(h_{\iota}\right)\right|_{\mathfrak{n}_{\Theta}^{-}}\right)$converges to a linear transformation $\tau: \mathfrak{n}_{\Theta}^{-} \rightarrow \mathfrak{n}_{\Theta}^{-}$with eigenvalues $a_{\alpha}=\lim _{\iota} \lambda_{-\alpha}\left(h_{\iota}\right), \alpha \in \Pi^{+}-\langle\Theta\rangle^{+}$(cf. [22, Proposition 2.5]). For a given $x \in v^{-1} N_{\Theta}^{-} b_{\Theta}^{+}$, it follows that $\left(g_{\iota} x\right)$ converges to a point in $u \exp (\operatorname{im}(\tau)) b_{\Theta}^{+}$. The connected sets $\operatorname{dom}_{\Theta}\left(g_{\iota}\right)=v^{-1}\left(N_{\Theta}^{-} b_{\Theta}^{+}\right)_{0}$ and $\operatorname{im}_{\Theta}\left(g_{\iota}\right)=u \exp (\operatorname{im}(\tau)) b_{\Theta}^{+}$are, respectively, called principal domain and principal image of $\left(g_{\iota}\right)$ in $\mathbb{F}_{\Theta}$. The principal image of $\left(g_{\iota}\right)$ reduces to a single point if and only if $\tau=0$, that is, $\lim _{\iota} \lambda_{-\alpha}\left(h_{\iota}\right)=0$ for every $\alpha \in \Pi^{+}-\langle\Theta\rangle^{+}$. In this case, the net $\left(g_{\iota}\right)$ is said to be contractible with respect to $\mathbb{F}_{\Theta}$.

In general, if $\Delta \subset \Theta \subset \Sigma$ and $\left(g_{\iota}\right)$ is a net in $G$, then $\pi_{\Theta}^{\Delta}\left(\operatorname{im}_{\Delta}\left(g_{\iota}\right)\right)=\operatorname{im}_{\Theta}\left(g_{\iota}\right)$.

Lemma 4.1 Let $g_{\iota}=u_{\iota} h_{\iota} v_{\iota}$ be a contractible net with respect to $\mathbb{F}_{\Theta}$, with $u_{\iota} \rightarrow u$ and $v_{\iota} \rightarrow 1$. Assume that $C \subset N^{-} b_{\Theta}^{+}$is a compact subset and consider $b \neq u b_{\Theta}^{+}$. There exists $\iota_{0} \in \mathcal{I}$ such that $g_{\iota}^{-1} b \notin C$ for all $\iota \geq \iota_{0}$.

Proof Repeat the proof for Lemma 6.1 of [3] by considering net instead of sequence.

For a given point $x \in B$ and a divergent net $t_{\iota} \rightarrow_{\mathcal{F}} \infty$ in $S$, assume that the net $\left(t_{l} x\right)$ converges to a point $y \in B$. Pick $i, j \in I$ such that $x \in U_{i}$ and $y \in U_{j}$, and take local sections $\chi_{i}: U_{i} \rightarrow Q$ and $\chi_{j}: U_{j} \rightarrow Q$. Let $\rho_{i j}$ be a local cocycle determined by $\chi_{i}$ and $\chi_{j}$ and consider the polar decomposition $g_{\iota}=u_{\iota} h_{\iota} v_{\iota}$ of the net $g_{\iota}=\rho_{i j}\left(t_{\iota}, x\right)$ in $G$.

Lemma 4.2 For a given $x \in B$, let the hypothesis and notations be as in the last paragraph above. For every set $\Theta \subset \Sigma$, one has $\chi_{j}(y) \cdot \operatorname{im}_{\Theta}\left(g_{\iota}\right) \subset E_{\Theta}$ (1). In particular, if $\lim _{\iota} e^{-\alpha\left(\log h_{\iota}\right)} \neq 0$, for every $\alpha \in \Pi^{+}-\langle\Theta\rangle^{+}$, then the action of $S$ on $\mathbb{E}_{\Theta}$ is $\mathcal{F}$-chain transitive.

Proof For $\mathcal{U}_{\varepsilon} \in \mathcal{O}_{\Theta}$ and $A \in \mathcal{F}$, we have $\mathbb{D}_{\mathcal{U}_{\varepsilon}, A}^{\Theta}(1)_{0} \cap\left(\mathbb{E}_{\Theta}\right)_{x} \neq \emptyset$. Since $\operatorname{dom}_{\Theta}\left(g_{\iota}\right)$ is dense in $\mathbb{F}_{\Theta}, \chi_{i}(x) \cdot \operatorname{dom}_{\Theta}\left(g_{\iota}\right)$ is dense in the fiber $\left(\mathbb{E}_{\Theta}\right)_{x}$ over $x$. As $\mathbb{D}_{\mathcal{U}_{\varepsilon}, A}^{\Theta}(1)_{0}$ is open, it follows that $\mathbb{D}_{\mathcal{U}_{\varepsilon}, A}^{\Theta}(1)_{0} \cap \chi_{i}(x) \cdot \operatorname{dom}_{\Theta}\left(g_{\iota}\right) \neq \emptyset$. Now, if $\chi_{i}(x) \cdot z \in \mathbb{D}_{\mathcal{U}_{\varepsilon}, A}^{\Theta}(1)_{0} \cap \chi_{i}(x) \cdot \operatorname{dom}_{\Theta}\left(g_{\iota}\right)$, then

$$
t_{\iota}\left(\chi_{i}(x) \cdot z\right)=t_{\iota} \chi_{i}(x) \cdot z=\chi_{j}\left(t_{\iota} x\right) \rho_{i j}\left(t_{\iota}, x\right) \cdot z=\chi_{j}\left(t_{\iota} x\right) \cdot g_{\iota} z
$$

and hence $t_{\iota}\left(\chi_{i}(x) \cdot z\right) \rightarrow \chi_{j}(y) \cdot z^{\prime}$ with $z^{\prime} \in \operatorname{im}_{\Theta}\left(g_{l}\right)$. This means that

$$
\chi_{j}(y) \cdot z^{\prime} \in \omega\left(\chi_{i}(x) \cdot z, \mathcal{F}\right) \cap \chi_{j}(y) \cdot \operatorname{im}_{\Theta}\left(g_{\iota}\right)
$$

and therefore $\chi_{j}(y) \cdot z^{\prime}$ is $\mathcal{F}$-chain recurrent (Remark 3.4). However, as $\mathbb{D}_{\mathcal{U}_{\varepsilon}, A}^{\Theta}(1)$ is the invariant control set of $\operatorname{End}_{l}\left(\mathbb{E}_{\Theta}\right)_{\mathcal{U}_{\varepsilon}, A}$, we have

$$
\omega\left(\chi_{i}(x) \cdot z, \mathcal{F}\right) \subset \operatorname{cls}\left(A \chi_{i}(x) \cdot z\right) \subset \operatorname{cls}\left(\mathbb{D}_{\mathcal{U}_{\varepsilon}, A}^{\Theta}(1)\right)
$$

and then $\chi_{j}(y) \cdot z^{\prime} \in E_{\Theta}(1)$. Thus, $\chi_{j}(y) \cdot \operatorname{im}_{\Theta}\left(g_{\iota}\right)$ meets $E_{\Theta}(1)$. By similar way, we can see that each point of $\chi_{j}(y) \cdot \operatorname{im}_{\Theta}\left(g_{l}\right)$ belongs to the $\omega$-limit set of some point in $\chi_{i}(x) \cdot \operatorname{dom}_{\Theta}\left(g_{l}\right)$; hence, $\chi_{j}(y) \cdot \operatorname{im}_{\Theta}\left(g_{\imath}\right)$ is $\mathcal{F}$-chain recurrent. Since $\chi_{j}(y) \cdot \operatorname{im}_{\Theta}\left(g_{\iota}\right)$ is connected and is contained in the fiber $\left(\mathbb{E}_{\Theta}\right)_{y}$ over $y$, it follows that $\chi_{j}(y) \cdot \operatorname{im}_{\Theta}\left(g_{l}\right)$ is $\mathcal{F}$-chain transitive $(\operatorname{Remark} 3.3)$. As $\chi_{j}(y) \cdot \operatorname{im}_{\Theta}\left(g_{\iota}\right)$ meets $E_{\Theta}(1)$, this means that $\chi_{j}(y) \cdot \operatorname{im}_{\Theta}\left(g_{l}\right) \subset$ $E_{\Theta}$ (1). Now, suppose that $\lim _{\iota} \mathrm{e}^{-\alpha\left(\log h_{l}\right)} \neq 0$ for every $\alpha \in \Pi^{+}-\langle\Theta\rangle^{+}$. Since the linear map $\tau=\lim _{\iota}\left(\left.\operatorname{Ad}\left(h_{\iota}\right)\right|_{\mathfrak{n}_{\Theta}^{-}}\right)$has eigenvalues $a_{\alpha}=\lim _{\iota} \mathrm{e}^{-\alpha\left(\log h_{\iota}\right)}$, it follows that $\tau$ is bijective. Moreover, as $\mathfrak{n}_{\Theta}^{-}$is nilpotent, exp $\left.\right|_{\mathfrak{n}_{\Theta}^{-}}$is surjective, and then $\operatorname{im}_{\Theta}\left(g_{\iota}\right)=u \exp (\operatorname{im} \tau) b_{\Theta}^{+}=$ $u N_{\Theta}^{-} b_{\Theta}^{+}$. By the first part of the proof, we have 


$$
\begin{aligned}
\chi_{j}(y) \cdot \operatorname{im}_{\Theta}\left(g_{\iota}\right) & \subset E_{\Theta}(1) \cap\left(\mathbb{E}_{\Theta}\right)_{y}=\bigcap_{\mathcal{U}_{\varepsilon} \in \mathcal{O}_{\Theta}, A \in \mathcal{F}} \mathbb{D}_{\mathcal{U}_{\varepsilon}, A}^{\Theta}(1)_{0} \cap\left(\mathbb{E}_{\Theta}\right)_{y} \\
& =\bigcap_{\mathcal{U}_{\varepsilon} \in \mathcal{O}_{\Theta}, A \in \mathcal{F}} \chi_{j}(y) \cdot \mathbb{A}_{\mathcal{U}_{\varepsilon}, A}^{\Theta}(1)_{0}
\end{aligned}
$$

and then $\operatorname{im}_{\Theta}\left(g_{\iota}\right) \subset \mathbb{A}_{\mathcal{U}_{\varepsilon}, A}^{\Theta}(1)_{0}$ for every induced subsemigroup $\left(\mathcal{S}_{\mathcal{U}_{\varepsilon}, A}\right)_{\chi_{j}(y)}$ on $\mathbb{F}_{\Theta}\left(\mathcal{U}_{\varepsilon} \in\right.$ $\mathcal{O}_{\Theta}$ and $\left.A \in \mathcal{F}\right)$. Hence, $u N_{\Theta}^{-} b_{\Theta}^{+} \subset \mathbb{A}_{\mathcal{U}_{\varepsilon}, A}^{\Theta}(1)_{0}$, and since $u N_{\Theta}^{-} b_{\Theta}^{+}$is dense in $\mathbb{F}_{\Theta}$ and $\mathbb{A}_{\mathcal{U}_{\varepsilon}, A}^{\Theta}(1)_{0}$ is dense in $\mathbb{A}_{\mathcal{U}_{\varepsilon}, A}^{\Theta}(1)$, we have $\mathbb{A}_{\mathcal{U}_{\varepsilon}, A}^{\Theta}(1)=\mathbb{F}_{\Theta}$ for all $\mathcal{U}_{\varepsilon} \in \mathcal{O}_{\Theta}$ and $A \in \mathcal{F}$. This means that $\mathbb{A}_{\mathcal{U}_{\varepsilon}, A}^{\Theta}(w)=\mathbb{A}_{\mathcal{U}_{\varepsilon}, A}^{\Theta}(1)$, for every $w \in W$, and then $\mathbb{D}_{\mathcal{U}_{\varepsilon}, A}^{\Theta}(w)=\mathbb{D}_{\mathcal{U}_{\varepsilon}, A}^{\Theta}(1)$ for every $w \in W$. Therefore, $E_{\Theta}(w)=E_{\Theta}$ (1) for every $w \in W$. Since the control sets $\mathbb{D}_{\mathcal{U}_{\varepsilon}, A}^{\Theta}(1)$ do not depend on the points of $Q$, we have $\mathbb{A}_{\mathcal{U}_{\varepsilon}, A}^{\Theta}(w)=\mathbb{A}_{\mathcal{U}_{\varepsilon}, A}^{\Theta}$ (1) for every induced subsemigroup $\left(\mathcal{S}_{\mathcal{U}_{\varepsilon}, A}\right)_{q}$ with $q \in Q$. As $\mathbb{A}_{\mathcal{U}_{\varepsilon}, A}^{\Theta}\left(w_{0}\right)_{0}=\mathbb{A}_{\mathcal{U}_{\varepsilon}, A}^{\Theta}(1)_{0}^{-}$, where $\mathbb{A}_{\mathcal{U}_{\varepsilon}, A}^{\Theta}(1)^{-}$is the invariant control set of $\left(\mathcal{S}_{\mathcal{U}_{\varepsilon}, A}\right)_{q}^{-1}$, it follows that $\mathbb{A}_{\mathcal{U}_{\varepsilon}, A}^{\Theta}(1)_{0}^{-}=\mathbb{A}_{\mathcal{U}_{\varepsilon}, A}^{\Theta}(1)_{0}$, which means that $\left(\mathcal{S}_{\mathcal{U}_{\varepsilon}, A}\right)_{q}$ is transitive on $\mathbb{F}_{\Theta}\left[21\right.$, Corollary 2.1]. But this is possible only if $\left(\mathcal{S}_{\mathcal{U}_{\varepsilon}, A}\right)_{q}=G$ or $\mathbb{F}_{\Theta}$ is trivial [21, Theorem 6.2]. In any case, we have $\mathbb{A}_{\mathcal{U}_{\varepsilon}, A}^{\Theta}(1)=\mathbb{F}_{\Theta}$, hence

$$
E_{\Theta}(1) \cap\left(\mathbb{E}_{\Theta}\right)_{\pi(q)}=q \cdot \mathbb{F}_{\Theta}
$$

for every $q \in Q$, and therefore $E_{\Theta}(1)=\mathbb{E}_{\Theta}$.

Lemma 4.3 Let $x \in B$ and $t_{\iota} \rightarrow \mathcal{F} \infty$ in $S$. Keep the notations as above. If the net $\left(t_{l} x\right)$ converges to some point $y$ in $B$, then the principal image $\operatorname{im}_{\Theta(\sigma)}\left(g_{l}\right)$ reduces to a single point.

Proof Let $\Theta_{\mathcal{U}_{\varepsilon_{0}}, A_{0}}=\Theta(\sigma)$ as in Lemma 3.1. Since $\mathbb{D}_{\mathcal{U}_{\varepsilon_{0}} A_{0}}^{\Theta(\sigma)}(1)_{0} \cap\left(\mathbb{E}_{\Theta(\sigma)}\right)_{y}=\chi_{j}(y)$. $\mathbb{A}_{\mathcal{U}_{\varepsilon_{0}}, A_{0}}^{\Theta(\sigma)}(1)_{0}$, Lemma 4.2 implies

$$
\chi_{j}(y) \cdot \operatorname{im}_{\Theta(\sigma)}\left(g_{t}\right) \subset E_{\Theta(\sigma)}(1) \cap\left(\mathbb{E}_{\Theta(\sigma)}\right)_{y} \subset \chi_{j}(y) \cdot \mathbb{A}_{\mathcal{U}_{\varepsilon_{0}}, t_{0}}^{\Theta(\sigma)}(1)_{0} .
$$

Hence, $\operatorname{im}_{\Theta(\sigma)}\left(g_{\iota}\right) \subset \mathbb{A}_{\mathcal{U}_{\varepsilon_{0}}, A_{0}}^{\Theta(\sigma)}(1)_{0}$. Since $\mathbb{A}_{\mathcal{U}_{\varepsilon_{0}}, A_{0}}^{\Theta(\sigma)}(1)$ is the invariant control set of $\left(\mathcal{S}_{\mathcal{U}_{\varepsilon_{0}}, A_{0}}\right)_{\chi_{j}(y)}, \mathbb{A}_{\mathcal{U}_{\varepsilon_{0}}, A_{0}}^{\Theta(\sigma)}(1)$ is contained in some open Bruhat cell (cf. [3, Proposition 5.4]). But the only possibility for this occurrence is when $\left(g_{l}\right)$ is contractible with respect to $\Theta(\sigma)$, that is, $\operatorname{im}_{\Theta(\sigma)}\left(g_{\iota}\right)$ reduces to a single point.

The following result plays a fundamental role in the algebraic characterization of the maximal chain transitive sets in the flag bundle.

Proposition 4.1 Assume that $x \in B$ is such that $\omega(x, \mathcal{F}) \neq \emptyset$. Then, $E_{\Theta(\sigma)}(1)$ meets the fiber $\left(\mathbb{E}_{\Theta(\sigma)}\right)_{x}$ over $x$ in a single point.

Proof Let $\Theta_{\mathcal{U}_{\varepsilon_{0}}, A_{0}}=\Theta(\sigma)$ and take $i \in I$ such that $x \in U_{i}$. We have

$$
E_{\Theta(\sigma)}(1) \cap\left(\mathbb{E}_{\Theta(\sigma)}\right)_{x}=\chi_{i}(x) \cdot \bigcap_{\mathcal{U}_{\varepsilon} \in \mathcal{O}_{\Theta}, A \in \mathcal{F}} \mathbb{A}_{\mathcal{U}_{\varepsilon}, A}^{\Theta(\sigma)}(1)_{0}
$$

where $\mathbb{A}_{\mathcal{U}_{\varepsilon}, A}^{\Theta(\sigma)}(1)$ is the invariant control set of $\left(\mathcal{S}_{\mathcal{U}_{\varepsilon}, A}\right)_{\chi_{i}(x)}$ in $\mathbb{F}_{\Theta(\sigma)}$. Set $\mathbb{A}=\bigcap_{\mathcal{U}_{\varepsilon}, A} \mathbb{A}_{\mathcal{U}_{\varepsilon}, A}^{\Theta(\sigma)}(1)_{0}$ and take $b_{0}=b_{\Theta(\sigma)}^{+} \in \mathbb{A}$. As $E_{\Theta(\sigma)}(1) \cap\left(\mathbb{E}_{\Theta(\sigma)}\right)_{x}$ is compact, it follows that $\mathbb{A}$ is a compact subset of $\mathbb{F}_{\Theta(\sigma)}$. Let $G=K \operatorname{cls}\left(A^{+}\right) K$ be the polar decomposition, assuming that $A^{+}$is a 
Weyl chamber that meets $\left(\mathcal{S}_{\mathcal{U}_{\varepsilon_{0}}, A_{0}}\right)_{\chi_{i}(x)}\left[17\right.$, Lemma 3.2]. We have $\mathbb{A}_{\mathcal{U}_{\varepsilon_{0}}, A_{0}}^{\Theta(\sigma)}(1) \subset N_{\Theta(\sigma)}^{-} b_{0}$ [3, Proposition 5.4], and then $\mathbb{A} \subset N_{\Theta(\sigma)}^{-} b_{0}$. For a given $y=\lim _{t_{l} \rightarrow \mathcal{F} \infty} t_{l} x \in \omega(x, \mathcal{F})$, take $j \in I$ such that $y \in U_{j}$ and consider a local cocycle $\rho_{i j}$ determined by the local sections $\chi_{i}$ and $\chi_{j}$. Denote $g_{\iota}=\rho_{i j}\left(t_{\iota}, x\right)$ and write $g_{\iota}=u_{\iota} h_{\iota} v_{\iota}$, where $u_{\iota}, v_{\iota} \in K$ and $h_{\iota} \in \operatorname{cls}\left(A^{+}\right)$. We may assume that $u_{\iota} \rightarrow u$ and $v_{\iota} \rightarrow v$ in $K$. Moreover, by defining $\chi_{i}^{\prime}\left(x^{\prime}\right)=\chi_{i}\left(x^{\prime}\right) v^{-1}$ for every $x^{\prime} \in U_{i}$, we obtain a local section $\chi_{i}^{\prime}: U_{i} \rightarrow Q$ such that $t_{\iota} \chi_{i}^{\prime}(x)=\chi_{j}\left(t_{\iota} x\right) g_{\iota} v^{-1}$ for all $\iota \geq \iota_{0}$. Then, we may assume that $v_{\iota} \rightarrow 1$. By Lemma 4.3, the net $\left(g_{\iota}\right)$ is contractible in $\mathbb{F}_{\Theta(\sigma)}$, that is, $\operatorname{im}_{\Theta(\sigma)}\left(g_{\iota}\right)$ reduces to the point $u b_{0}$. By Lemma 4.1, it follows that $b \neq u b_{0}$; hence, there is $\iota_{0}$ such that $g_{\iota}^{-1} b \notin \mathbb{A}$ for all $\iota \geq i_{0}$. By the cocycle property, we have

$$
t_{\iota}^{-1}\left(\chi_{j}\left(t_{\iota} x\right) \cdot b\right)=\chi_{i}(x) \rho_{j i}\left(t_{\iota}^{-1}, t_{\iota} x\right) \cdot b=\chi_{i}(x) \cdot g_{\iota}^{-1} b
$$

for every $\iota$ such that $t_{\iota} x \in U_{j}$. Hence, for every $\iota \geq i_{0}$ with $t_{\iota} x \in U_{j}$, we have

$$
\chi_{i}(x) \cdot g_{\iota}^{-1} b \notin \chi_{i}(x) \cdot \mathbb{A}=E_{\Theta(\sigma)}(1) \cap\left(\mathbb{E}_{\Theta(\sigma)}\right)_{x} .
$$

As $\chi_{i}(x) \cdot g_{\iota}^{-1} b \in\left(\mathbb{E}_{\Theta(\sigma)}\right)_{x}$, it follows that $\chi_{i}(x) \cdot g_{\iota}^{-1} b \notin E_{\Theta(\sigma)}$ (1), that is, $t_{l}^{-1}\left(\chi_{j}\left(t_{l} x\right) \cdot b\right) \notin E_{\Theta(\sigma)}(1)$. Since $E_{\Theta(\sigma)}(1)$ is invariant by $S^{-1}$, we have $\chi_{j}\left(t_{\iota} x\right) \cdot b \notin$ $E_{\Theta(\sigma)}(1)$. Hence, the intersection $E_{\Theta(\sigma)}(1) \cap\left(\mathbb{E}_{\Theta(\sigma)}\right)_{t_{l} x}$ reduces to the point $\chi_{j}\left(t_{l} x\right) \cdot u b_{0}$. Now, since $E_{\Theta(\sigma)}(1)$ is invariant by $S$, by taking $\chi_{i}(x) \cdot b \in E_{\Theta(\sigma)}(1) \cap\left(\mathbb{E}_{\Theta(\sigma)}\right)_{x}$, we have $t_{l}\left(\chi_{i}(x) \cdot b\right) \in E_{\Theta(\sigma)}(1) \cap\left(\mathbb{E}_{\Theta(\sigma)}\right)_{t_{\iota} x}$, and then $t_{\iota}\left(\chi_{i}(x) \cdot b\right)=\chi_{j}\left(t_{l} x\right) \cdot u b_{0}$. As $\sigma_{t_{l}}^{\mathbb{E}_{\Theta(\sigma)}}$ is a homeomorphism between the fibers $\left(\mathbb{E}_{\Theta(\sigma)}\right)_{x}$ and $\left(\mathbb{E}_{\Theta(\sigma)}\right)_{t_{l} x}$, we conclude that $E_{\Theta(\sigma)}(1) \cap\left(\mathbb{E}_{\Theta(\sigma)}\right)_{x}$ is a single point.

Let $t_{\iota} \rightarrow \mathcal{F} \infty$ and assume that the net $\left(t_{l}^{-1} x\right)$ converges to $z \in B$. Take $i, j \in I$ such that $z \in U_{j}$ and $x \in U_{i}$, and then define the net $g_{\iota}=\rho_{i j}\left(t_{\iota}^{-1}, x\right)$ in $G$. Consider the polar decomposition $g_{\iota}=u_{\iota} h_{\iota} v_{\iota}$.

Lemma 4.4 For $x \in B$, let the hypothesis and notation be as in the last paragraph above. For a given set $\Theta \subset \Sigma, \chi_{j}(z) \cdot \operatorname{im}_{\Theta}\left(g_{\iota}\right) \subset E_{\Theta}\left(w_{0}\right)$. In particular, if $\lim _{\iota} e^{-\alpha\left(\log h_{\iota}\right)} \neq 0$, for every $\alpha \in \Pi^{+}-\langle\Theta\rangle^{+}$, then the action of $S$ on $\mathbb{E}_{\Theta}$ is $\mathcal{F}$-chain transitive.

Proof If $\chi_{i}(x) \cdot v \in \chi_{i}(x) \cdot \operatorname{dom}_{\Theta}\left(g_{\imath}\right) \cap \mathbb{D}_{\mathcal{U}_{\varepsilon}, A}^{\Theta}\left(w_{0}\right)_{0}$, then $t_{l}^{-1}\left(\chi_{i}(x) \cdot v\right) \rightarrow \chi_{j}(z) \cdot v^{\prime}$, where $v^{\prime} \in \operatorname{im}_{\Theta}\left(g_{\iota}\right)$. Hence, $\chi_{j}(z) \cdot v^{\prime} \in \omega^{*}\left(\chi_{i}(x) \cdot v, \mathcal{F}\right) \cap \chi_{j}(z) \cdot \operatorname{im}_{\Theta}\left(g_{l}\right)$, and therefore $\chi_{j}(z) \cdot v^{\prime}$ is $\mathcal{F}$-chain recurrent. Suppose that $\chi_{j}(z) \cdot v^{\prime} \in E_{\Theta}(w)$ for some $w \in W$. Then, we have

$$
\begin{aligned}
\chi_{j}(z) \cdot v^{\prime} & \in \omega^{*}\left(\chi_{i}(x) \cdot v, \mathcal{F}\right) \cap \mathbb{D}_{\mathcal{U}_{\varepsilon}, A}^{\Theta}\left(w_{0}\right) \\
& \subset \Omega^{*}\left(\chi_{i}(x) \cdot v, \mathcal{F}\right) \cap \mathbb{D}_{\mathcal{U}_{\varepsilon}, A}^{\Theta}\left(w_{0}\right) \\
& \subset \operatorname{End}_{l}(\mathbb{E})_{\mathcal{U}_{\varepsilon}, A}^{*} \chi_{i}(x) \cdot v \cap \mathbb{D}_{\mathcal{U}_{\varepsilon}, A}^{\Theta}\left(w_{0}\right)
\end{aligned}
$$

and then $\chi_{i}(x) \cdot v \in \operatorname{End}_{l}(\mathbb{E})_{\mathcal{U}_{\varepsilon}, A} \chi_{j}(z) \cdot v^{\prime} \cap \mathbb{D}_{\mathcal{U}_{\varepsilon}, A}^{\Theta}\left(w_{0}\right)$. This means that $\chi_{j}(z) \cdot v^{\prime} \in$ $\mathbb{D}_{\mathcal{U}_{\varepsilon}, A}^{\Theta}(w) \cap \mathcal{A}\left(\mathbb{D}_{\mathcal{U}_{\varepsilon}, A}^{\Theta}\left(w_{0}\right)\right)$, that is, $\mathbb{D}_{\mathcal{U}_{\varepsilon}, A}^{\Theta}(w) \preceq \mathbb{D}_{\mathcal{U}_{\varepsilon}, A}^{\Theta}\left(w_{0}\right)$. Since $\mathbb{D}_{\mathcal{U}_{\varepsilon}, A}^{\Theta}\left(w_{0}\right)$ is the minimal effective control set of $\operatorname{End}_{l}(\mathbb{E})_{\mathcal{U}_{\varepsilon}, A}$, it follows that $\mathbb{D}_{\mathcal{U}_{\varepsilon}, A}^{\Theta}(w)=\mathbb{D}_{\mathcal{U}_{\varepsilon}, A}^{\Theta}\left(w_{0}\right)$, and therefore $E_{\Theta}(w)=E_{\Theta}\left(w_{0}\right), \omega^{*}\left(\chi_{i}(x) \cdot v, \mathcal{F}\right) \subset E_{\Theta}\left(w_{0}\right)$, and $\chi_{j}(z) \cdot v^{\prime} \in$ $E_{\Theta}\left(w_{0}\right) \cap \chi_{j}(z) \cdot \operatorname{im}_{\Theta}\left(g_{l}\right)$. However, each point in $\chi_{j}(z) \cdot \operatorname{im}_{\Theta}\left(g_{\imath}\right)$ is contained in the $\omega^{*}$-limit set of some point of $\chi_{i}(x) \cdot \operatorname{dom}_{\Theta}\left(g_{\imath}\right)$. Hence, $\chi_{i}(x) \cdot \operatorname{dom}_{\Theta}\left(g_{l}\right)$ is $\mathcal{F}$-chain recurrent and connected, which implies that $\chi_{j}(z) \cdot \operatorname{im}_{\Theta}\left(g_{\iota}\right) \subset E_{\Theta}\left(w_{0}\right)$. Now, by assuming that 
$\lim _{\iota} \mathrm{e}^{-\alpha\left(\log h_{\iota}\right)} \neq 0$, for every $\alpha \in \Pi^{+}-\langle\Theta\rangle^{+}$, repeat the proof for Lemma 4.2 to show that the action of $S$ on $\mathbb{E}_{\Theta}$ is $\mathcal{F}$-chain transitive.

Proposition 4.2 Let $x \in B$ and $t_{\iota} \rightarrow \mathcal{F} \infty$ in $S$. Keep the notations as above. If the net $\left(t_{\iota}^{-1} x\right)$ converges to some point $z$ in $B$, then the principal image $\operatorname{im}_{\Theta *}(\sigma)\left(g_{\iota}\right)$ reduces to a single point.

Proof Take $\Theta_{\mathcal{U}_{\varepsilon_{0}}, A_{0}}=\Theta(\sigma)$ as in Lemma 3.1. Since $\Theta^{*}(\sigma)$ is the parabolic type of the $\operatorname{semigroup}\left(\mathcal{S}_{\mathcal{U}_{\varepsilon_{0}}, A_{0}}\right)_{\chi_{j}(z)}^{-1}$, we have $\mathbb{A}_{\mathcal{U}_{\varepsilon_{0}}, A_{0}}^{\Theta^{*}(\sigma)}\left(w_{0}\right)_{0}=\mathbb{A}_{\mathcal{U}_{\varepsilon_{0}}, A_{0}}^{\Theta^{*}(\sigma)}(1)_{0}^{-}$. Then, Lemma 4.4 implies

$$
\chi_{j}(z) \cdot \operatorname{im}_{\Theta^{*}(\sigma)}\left(g_{\iota}\right) \subset E_{\Theta^{*}(\sigma)}\left(w_{0}\right) \cap\left(\mathbb{E}_{\Theta^{*}(\sigma)}\right)_{z} \subset \chi_{j}(z) \cdot \mathbb{A}_{\mathcal{U}_{\varepsilon_{0}}}^{\Theta^{*}(\sigma)}(1)_{0}^{-},
$$

hence $\operatorname{im}_{\Theta^{*}(\sigma)}\left(h_{\iota}\right) \subset \mathbb{A}_{\mathcal{U}_{\varepsilon_{0}}, A_{0}}^{\Theta^{*}(\sigma)}(1)_{0}^{-}$. Since $\mathbb{A}_{\mathcal{U}_{\varepsilon_{0}}, A_{0}}^{\Theta^{*}(\sigma)}(1)^{-}$is contained in some open Bruhat cell, it follows that $\operatorname{im}_{\Theta^{*}(\sigma)}\left(h_{l}\right)$ is a single point.

Proposition 4.3 Assume that $x \in B$ is such that $\omega^{*}(x, \mathcal{F}) \neq \emptyset$. Then, $E_{\Theta^{*}(\sigma)}\left(w_{0}\right)$ meets the fiber $\left(\mathbb{E}_{\Theta(\sigma)}\right)_{x}$ over $x$ in a single point.

Proof Let $\Theta_{\mathcal{U}_{\varepsilon_{0}}, A_{0}}=\Theta(\sigma)$ and take $i \in I$ such that $x \in U_{i}$. We have

$$
E_{\Theta^{*}(\sigma)}\left(w_{0}\right) \cap\left(\mathbb{E}_{\Theta^{*}(\sigma)}\right)_{x}=\chi_{i}(x) \cdot \bigcap_{\mathcal{U}_{\varepsilon} \in \mathcal{O}_{\Theta^{*}(\sigma)}, A \in \mathcal{F}} \mathbb{A}_{\mathcal{U}_{\varepsilon}, A}^{\Theta^{*}(\sigma)}(1)_{0}^{-} .
$$

Set $\mathbb{A}^{-}=\bigcap_{\mathcal{U}_{\varepsilon}, A} \mathbb{A}_{\mathcal{U}_{\varepsilon}, A}^{\Theta^{*}(\sigma)}(1)_{0}^{-}$and take $b_{0}=\mathfrak{p}_{\Theta^{*}(\sigma)}(\mu) \in \mathbb{A}^{-}$, where the Weyl chamber $\mu$ meets $\left(\mathcal{S}_{\mathcal{U}_{\varepsilon_{0}}, A_{0}}\right)_{\chi_{i}(x)}^{-1}$. Let $G=K \operatorname{cls}\left(A^{+}(\mu)\right) K$ be the polar decomposition of $G$ determined by $\mathfrak{a}^{+}(\mu)$. Since $\Theta^{*}(\sigma)=\Theta\left(\left(\mathcal{S}_{\mathcal{U}_{\varepsilon_{0}}, A_{0}}\right)_{\chi_{i}(x)}^{-1}\right)$, we have $\mathbb{A}_{\mathcal{U}_{\varepsilon_{0}}, A_{0}}^{\Theta^{*}(\sigma)}(1)^{-} \subset N_{\Theta *(\sigma)}^{-} b_{0}$. Hence, $\mathbb{A}^{-} \subset N_{\Theta^{*}(\sigma)}^{-} b_{0}$. For a given $z=\lim _{t_{l} \rightarrow \mathcal{F} \infty} t_{l}^{-1} x \in \omega^{*}(x, \mathcal{F})$, take $j \in I$ such that $y \in U_{j}$ and consider a local cocycle $\rho_{i j}$ determined by the local sections $\chi_{i}$ and $\chi_{j}$. Denote $g_{\iota}=\rho_{i j}\left(t_{\iota}^{-1}, x\right)$ and write $g_{\iota}=u_{\iota} h_{\iota} v_{\iota}$, where $u_{\iota}, v_{\iota} \in K$ and $h_{\iota} \in \operatorname{cls}\left(A^{+}(\mu)\right)$. By Proposition 4.2, $\operatorname{im}_{\Theta^{*}(\sigma)}\left(g_{\iota}\right)$ reduces to the point $u b_{0}$. By Lemma 4.1, it follows that $b \neq u b_{0}$, and then there is $\iota_{0}$ such that $g_{\iota}^{-1} b \notin \mathbb{A}^{-}$for all $\iota \geq \iota_{0}$. For every $t \in S$ with $t^{-1} x \in U_{j}$, we have

$$
t\left(\chi_{j}\left(t^{-1} x\right) \cdot b\right)=\chi_{i}(x) \rho_{j i}\left(t, t^{-1} x\right) \cdot b=\chi_{i}(x) \cdot h_{t}^{-1} b .
$$

Hence,

$$
\chi_{i}(x) \cdot h_{\imath}^{-1} b \notin \chi_{i}(x) \cdot \mathbb{A}^{-}=E_{\Theta^{*}(\sigma)}\left(w_{0}\right) \cap\left(\mathbb{E}_{\Theta^{*}(\sigma)}\right)_{x}
$$

for all $\iota \geq \iota_{0}$ such that $t_{\iota}^{-1} x \in U_{j}$. As $\chi_{i}(x) \cdot h_{\iota}^{-1} b \in\left(\mathbb{E}_{\Theta^{*}(\sigma)}\right)_{x}$, it follows that $\chi_{i}(x) \cdot h_{\iota}^{-1} b \notin$ $E_{\Theta^{*}(\sigma)}\left(w_{0}\right)$, that is, $t_{\iota}\left(\chi_{j}\left(t_{l}^{-1} x\right) \cdot b\right) \notin E_{\Theta^{*}(\sigma)}\left(w_{0}\right)$, and then $\chi_{j}\left(t_{\imath}^{-1} x\right) \cdot b \notin E_{\Theta^{*}(\sigma)}\left(w_{0}\right)$. Thus, the intersection $E_{\Theta^{*}(\sigma)}\left(w_{0}\right) \cap\left(\mathbb{E}_{\Theta^{*}(\sigma)}\right)_{t_{l}^{-1} x}$ reduces to the point $\chi_{j}\left(t_{l}^{-1} x\right) \cdot u b_{0}$. Finally, since $\sigma_{t_{l}}^{\mathbb{E}_{\Theta^{*}(\sigma)}}$ is a bijection between the fibers $\left(\mathbb{E}_{\Theta^{*}(\sigma)}\right)_{t_{l}^{-1} x}$ and $\left(\mathbb{E}_{\Theta *}(\sigma)\right)_{x}$, we conclude that $E_{\Theta *(\sigma)}\left(w_{0}\right) \cap\left(\mathbb{E}_{\Theta^{*}(\sigma)}\right)_{x}$ is a single point.

Note that $S=T=S^{-1} S$ whenever $S$ is a group. In this case, Lemmas 4.2 and 4.4 together imply $E_{\Theta}(1)=E_{\Theta}\left(w_{0}\right)$. Hence, there is a unique $\mathcal{F}$-chain transitive set in $\mathbb{E}_{\Theta}$, and therefore $\left(S, \mathbb{E}_{\Theta}, \sigma^{\mathbb{E}_{\Theta}}\right)$ is $\mathcal{F}$-chain transitive for every subset $\Theta \subset \Sigma$, since $E_{\Theta}(1) \cap\left(\mathbb{E}_{\Theta}\right)_{\pi(q)}=$ $q \cdot \mathbb{F}_{\Theta}$, for every $q \in Q$, and therefore $E_{\Theta}(1)=\mathbb{E}_{\Theta}$. 
Another question is about the relationship between the parabolic types of $S$ and $S^{-1}$. We can provide a relation in the case of compact base space. For the following, we define the reverse filter basis $\mathcal{F}^{-1}=\left\{A^{-1}: A \in \mathcal{F}\right\}$. For each $w \in W$, we denote by $E_{\Theta}^{-}(w)$ the maximal $\mathcal{F}^{-1}$-chain transitive set of $S^{-1}$ in $\mathbb{E}_{\Theta}$.

Proposition 4.4 Assume that the base space $B$ is compact. Then, the maximal $\mathcal{F}^{-1}$-chain transitive sets of $\left(S^{-1}, \mathbb{E}_{\Theta}\right)$ coincide with the maximal $\mathcal{F}$-chain transitive sets of $\left(S, \mathbb{E}_{\Theta}\right)$, inverting the dynamic order. In particular, $E_{\Theta}^{-}(1)=E_{\Theta}\left(w_{0}\right)$ and $E_{\Theta}^{-}\left(w_{0}\right)=E_{\Theta}$ (1).

Proof For any $\Theta \subset \Sigma$, the total space $\mathbb{E}_{\Theta}$ is compact Hausdorff, because the base space and the fibers are compact. Since the number of maximal $\mathcal{F}$-chain transitive sets of $\left(S, \mathbb{E}_{\Theta}\right)$ is finite, there exists the finest $\mathcal{F}$-Morse decomposition for $\left(S, \mathbb{E}_{\Theta}\right)$ [7, Theorem 5.2]. It follows that the maximal $\mathcal{F}$-chain transitive sets of $\left(S, \mathbb{E}_{\Theta}\right)$ coincide with the $\mathcal{F}$-Morse sets. An $\mathcal{F}$-Morse set is an intersection of an $\mathcal{F}$-attractor with an $\mathcal{F}$-repeller. An $\mathcal{F}$-attractor is an $\mathcal{F}^{-}$-repeller, while an $\mathcal{F}$-repeller is an $\mathcal{F}^{-}$-attractor. Hence, an $\mathcal{F}$-Morse set is an $\mathcal{F}^{-}$-Morse set, and vice versa. Thus, the maximal $\mathcal{F}$-chain transitive sets of $\left(S, \mathbb{E}_{\Theta}\right)$ coincide with the maximal $\mathcal{F}^{-1}$-chain transitive sets of $\left(S^{-1}, \mathbb{E}_{\Theta}\right)$. Moreover, the dynamic order among the $\mathcal{F}^{-}$-Morse sets is the reverse dynamic order among the $\mathcal{F}$-Morse sets. By Theorem 3.3, we have $E_{\Theta}^{-}(1)=E_{\Theta}\left(w_{0}\right)$ and $E_{\Theta}^{-}\left(w_{0}\right)=E_{\Theta}(1)$.

Then, we have the following result.

Proposition 4.5 If the base space B is compact, then $\Theta^{*}(\sigma)=\Theta\left(\sigma^{*}\right)$.

Proof Since $E\left(w_{0}\right)=E^{-}(1)$ and $E_{\Theta\left(\sigma^{*}\right)}\left(w_{0}\right)=E_{\Theta\left(\sigma^{*}\right)}^{-}(1)$, it follows by Proposition 3.2 that $\tilde{\pi}_{\Theta\left(\sigma^{*}\right)}^{-1}\left(E_{\Theta\left(\sigma^{*}\right)}\left(w_{0}\right)\right)=E\left(w_{0}\right)$, where $\tilde{\pi}_{\Theta\left(\sigma^{*}\right)}: \mathbb{E} \rightarrow \mathbb{E}_{\Theta\left(\sigma^{*}\right)}$ be the standard fibration. Let $\Theta(\sigma)=\Theta_{\mathcal{U}_{\varepsilon_{0}}, A_{0}}$. For a given $q \cdot u \in \bigcap_{\mathcal{U}_{\varepsilon}, A} q \cdot \mathbb{A}_{\mathcal{U}_{\varepsilon}, A}^{\Theta\left(\sigma^{*}\right)}(1)_{0}^{-} \subset E_{\Theta\left(\sigma^{*}\right)}\left(w_{0}\right)$, we have $\tilde{\pi}_{\Theta\left(\sigma^{*}\right)}^{-1}(q \cdot u) \subset q \cdot \mathbb{A}_{\mathcal{U}_{\varepsilon_{0}}, A_{0}}(1)_{0}^{-}$, and then $\pi_{\Theta\left(\sigma^{*}\right)}^{-1}(u) \subset \mathbb{A}_{\mathcal{U}_{\varepsilon_{0}}, A_{0}}(1)_{0}^{-}$. Now, if $v \in \mathbb{A}_{\mathcal{U}_{\varepsilon_{0}}, A_{0}}^{\Theta\left(\sigma^{*}\right)}(1)_{0}^{-}$, there is $g \in\left(\mathcal{S}_{\mathcal{U}_{\varepsilon_{0}}, A_{0}}\right)_{q}^{-1}$ such that $g u=v$. Then, $\pi_{\Theta\left(\sigma^{*}\right)}^{-1}(v)=$ $g \pi_{\Theta\left(\sigma^{*}\right)}^{-1}(u) \subset \mathbb{A}_{\mathcal{U}_{\varepsilon_{0}}, A_{0}}(1)_{0}^{-}$, because $\mathbb{A}_{\mathcal{U}_{\varepsilon_{0}}, A_{0}}(1)_{0}^{-}$is invariant by $\left(\mathcal{S}_{\mathcal{U}_{\varepsilon_{0}}, A_{0}}\right)_{q}^{-1}$. It follows that $\pi_{\Theta\left(\sigma^{*}\right)}^{-1}\left(\mathbb{A}_{\mathcal{U}_{\varepsilon_{0}}, A_{0}}^{\Theta\left(\sigma^{*}\right)}(1)_{0}^{-}\right) \subset \mathbb{A}_{\mathcal{U}_{\varepsilon_{0}}, A_{0}}(1)_{0}^{-}$, and therefore $\pi_{\Theta\left(\sigma^{*}\right)}^{-1}\left(\mathbb{A}_{\mathcal{U}_{\varepsilon_{0}}, A_{0}}^{\Theta\left(\sigma^{*}\right)}(1)_{0}^{-}\right)=$ $\mathbb{A}_{\mathcal{U}_{\varepsilon_{0}}, A_{0}}(1)_{0}^{-}$. This means that $\Theta\left(\sigma^{*}\right) \subset \Theta^{*}(\sigma)$. By considering the fibration $\tilde{\pi}_{\Theta(\sigma)}$ : $\mathbb{E} \rightarrow \mathbb{E}_{\Theta(\sigma)}$ and using similar argument, we can see that $\Theta(\sigma) \subset \Theta^{*}\left(\sigma^{*}\right)$, that is, $\Theta^{*}(\sigma) \subset \Theta\left(\sigma^{*}\right)$. Thus, $\Theta^{*}(\sigma)=\Theta\left(\sigma^{*}\right)$.

From now on, for every point $x \in B$, we assume that both limit sets $\omega(x, \mathcal{F})$ and $\omega^{*}(x, \mathcal{F})$ are nonempty. We shall describe the intersection of the maximal chain transitive sets with the fibers of the flag bundle. By Propositions 4.1 and 4.3, we have the following result.

Proposition 4.6 Both flag bundles $\mathbb{E}_{\Theta(\sigma)} \rightarrow B$ and $\mathbb{E}_{\Theta^{*}(\sigma)} \rightarrow B$ admit $T$-equivariant global cross sections.

Proof Define $\chi: B \rightarrow \mathbb{E}_{\Theta(\sigma)}$ such that $\chi(x)$ is the unique point in the intersection $E_{\Theta(\sigma)}(1) \cap\left(\mathbb{E}_{\Theta(\sigma)}\right)_{x}$ and define $\chi^{*}: B \rightarrow \mathbb{E}_{\Theta *(\sigma)}$ such that $\chi^{*}(x)$ is the unique point in the intersection $E_{\Theta *(\sigma)}\left(w_{0}\right) \cap\left(\mathbb{E}_{\Theta *(\sigma)}\right)_{\chi}$. By definition, we have $\pi_{\Theta(\sigma)} \circ \chi=I d_{B}$ and $\pi_{\Theta^{*}(\sigma)} \circ \chi^{*}=I d_{B}$. The graph of $\chi$ is $E_{\Theta(\sigma)}(1)$ and the graph of $\chi^{*}$ is $E_{\Theta^{*}(\sigma)}\left(w_{0}\right)$, which are closed sets (Remark 3.1). Since the fibers are compact, it follows that both $\chi$ 
and $\chi^{*}$ are continuous. Finally, since both $\mathbb{E}_{\Theta(\sigma)}$ and $\mathbb{E}_{\Theta *}(\sigma)$ are $T$-invariant, we have $E_{\Theta(\sigma)}(1) \cap\left(\mathbb{E}_{\Theta(\sigma)}\right)_{t x}=\{t \chi(x)\}$ and $E_{\Theta^{*}(\sigma)}\left(w_{0}\right) \cap\left(\mathbb{E}_{\Theta^{*}(\sigma)}\right)_{t x}=\left\{t \chi^{*}(x)\right\}$, for all $t \in T$ and $x \in B$, and hence $\chi(t x)=t \chi(x)$ and $\chi^{*}(t x)=t \chi^{*}(x)$. Therefore, both $\chi$ and $\chi^{*}$ are $T$-equivariant maps.

For each $q \in Q$, we write

$$
\chi(\pi(q))=\chi_{i_{q}}(\pi(q)) \cdot b_{q},
$$

where $\pi(q) \in U_{i_{q}}$ and $\chi$ is the cross section given in Proposition 4.6. Since $q=$ $\chi_{i_{q}}(\pi(q)) v_{i_{q}}(q)$, we have

$$
\chi(\pi(q))=q \cdot v_{i_{q}}(q)^{-1} b_{q} .
$$

Then, we denote by $q^{-1} \cdot \chi(\pi(q))$ the point $v_{i_{q}}(q)^{-1} b_{q} \in \mathbb{F}_{\Theta(\sigma)}$. Similarly, we define the point $q^{-1} \cdot \chi^{*}(\pi(q))$ in $\mathbb{F}_{\Theta^{*}(\sigma)}$. Thus, the global cross sections $\chi$ and $\chi^{*}$ are determined, respectively, by the maps $f: Q \rightarrow \mathbb{F}_{\Theta(\sigma)}$ and $f^{*}: Q \rightarrow \mathbb{F}_{\Theta^{*}(\sigma)}$ defined as

$$
f(q)=q^{-1} \cdot \chi(\pi(q)) \quad \text { and } \quad f^{*}(q)=q^{-1} \cdot \chi^{*}(\pi(q))
$$

(see [14]). For $g \in G$ and $q \in Q$, we have $\chi(\pi(q))=\chi(\pi(q g))$, and then

$$
f(q g)=v_{i_{q g}}(q g)^{-1} b_{q g}=g^{-1} v_{i_{q g}}(q)^{-1} b_{q g}=g^{-1} v_{i_{q}}(q)^{-1} b_{q}=g^{-1} f(q) .
$$

Similarly, we have $f^{*}(q g)=g^{-1} f^{*}(q)$. Thus, $f$ and $f^{*}$ are $G$-equivariant maps.

Since $\left(f(q), f^{*}(q)\right)=\left(v_{i_{q}}(q)^{-1} b_{q}, v_{i_{q}}(q)^{-1} b_{q}^{*}\right)$, with $b_{q} \in \mathbb{F}_{\Theta(\sigma)}$ and $b_{q}^{*} \in \mathbb{F}_{\Theta^{*}(\sigma)}$, the point $\left(f(q), f^{*}(q)\right)$ belongs to a $G$-orbit in $\mathbb{F}_{\Theta(\sigma)} \times \mathbb{F}_{\Theta^{*}(\sigma)}$. According to [3, Section 5.1], by taking

$$
\mathfrak{p}_{\Theta(\sigma)}=\mathfrak{p} \oplus \mathfrak{n}^{-}(\Theta(\sigma)) \in \mathbb{F}_{\Theta(\sigma)} \quad \text { and } \quad \mathfrak{p}_{\Theta(\sigma)}^{-}=\mathfrak{p}^{-} \oplus \mathfrak{n}(\Theta(\sigma)) \in \mathbb{F}_{\Theta *}(\sigma)
$$

where $\mathfrak{p}^{-}=\mathfrak{m} \oplus \mathfrak{a} \oplus \mathfrak{n}^{-}$, the orbit of $\left(\mathfrak{p}_{\Theta(\sigma)}, \mathfrak{p}_{\Theta(\sigma)}^{-}\right)$identifies with the adjoint orbit $\operatorname{Ad}(G) H_{\Theta(\sigma)}$, with $H_{\Theta(\sigma)} \in \operatorname{cls}\left(\mathfrak{a}^{+}\right)$such that $\Theta(\sigma)=\Theta\left(H_{\Theta(\sigma)}\right)=\left\{\alpha \in \Sigma: \alpha\left(H_{\Theta(\sigma)}\right)\right.$ $=0\}$. We define $h: Q \rightarrow \operatorname{Ad}(G) H_{\Theta(\sigma)}$ with $h(q) \in \operatorname{Ad}(G) H_{\Theta(\sigma)}$ the point identified with $\left(f(q), f^{*}(q)\right)$. Then, $h$ is continuous and equivariant, because $f$ and $f^{*}$ are continuous equivariant maps and $h(q g)=\operatorname{Ad}\left(g^{-1}\right) h(q)$, for all $q \in Q$ and $g \in G$. By defining $\zeta: B \rightarrow Q \times{ }_{G} \operatorname{Ad}(G) H_{\Theta(\sigma)}$ as

$$
\zeta(\pi(q))=q \cdot h(q),
$$

it is easily seen that $\zeta$ is a cross section for the associated bundle $Q \times{ }_{G} \operatorname{Ad}(G) H_{\Theta(\sigma)} \rightarrow B$. Since $Q \times{ }_{G} \operatorname{Ad}(G) H_{\Theta(\sigma)}$ is isomorphic with the quotient $Q / Z_{G}\left(H_{\Theta(\sigma)}\right)$, the principal bundle $(Q, \pi, B, G)$ can be reduced to the principal subbundle $\left(P, \pi, B, Z_{G}\left(H_{\Theta(\sigma)}\right)\right)$, where $P=\{q \in Q: \zeta(\pi(q))=\mu(q)\}$ and $\mu: Q \rightarrow Q / Z_{G}\left(H_{\Theta(\sigma)}\right)$ is the standard projection.

Proposition 4.7 The principal subbundle $\left(P, \pi, B, Z_{G}\left(H_{\Theta(\sigma)}\right)\right)$ is invariant by $T$.

Proof For $q \in Q$ and $t \in T$, we have $t \chi(\pi(q))=\chi(\pi(t q))$, because $E_{\Theta(\sigma)}(1)$ is invariant by $T$. Similarly, $t \chi^{*}(\pi(q))=\chi^{*}(\pi(t q))$. Hence, $f(t q)=f(q)$ and $f^{*}(t q)=f^{*}(q)$, and thus $h(t q)=h(q)$. It follows that

$$
\zeta(\pi(t q))=t q \cdot h(t q)=t q \cdot h(q)=t \zeta(\pi(q))
$$


for all $q \in Q$ and $t \in T$, and therefore $\zeta$ is $T$-equivariant. Now, for $q \in P$ and $t \in T$, we have

$$
\zeta(\pi(t q))=t \zeta(\pi(q))=t \mu(q)=\mu(t q)
$$

and therefore $P$ is $T$-invariant.

We now consider the semigroup of automorphisms $(S, P, \sigma)$. Since $Z_{G}\left(H_{\Theta(\sigma)}\right)$ is a reducible Lie group, we can induce actions of $S$ on fiber bundles associated with $\left(P, \pi, B, Z_{G}\left(H_{\Theta(\sigma)}\right)\right)$.

Writing $\Sigma=\left\{\alpha_{1}, \ldots, \alpha_{n}\right\}$, denote by $r_{i}$ the reflection with respect to the root $\alpha_{i}$ and by $\tilde{\pi}_{i}: \mathbb{E} \rightarrow \mathbb{E}_{\left\{\alpha_{i}\right\}}$ and $\pi_{i}: \mathbb{F} \rightarrow \mathbb{F}_{\left\{\alpha_{i}\right\}}$ the standard fibrations. Then, we define the exhausting map $\tilde{\gamma}_{i}$ between subsets of $\mathbb{E}$ as the set valued map

$$
\tilde{\gamma}_{i}(\mathbb{B})=\tilde{\pi}_{i}^{-1}\left(\tilde{\pi}_{i}(\mathbb{B})\right)
$$

for every subset $\mathbb{B} \subset \mathbb{E}$. The exhausting map $\gamma_{i}$ between subsets of the maximal flag $\mathbb{F}$ is given by

$$
\gamma_{i}(\mathbb{A})=\pi_{i}^{-1}\left(\pi_{i}(\mathbb{A})\right)
$$

for every subset $\mathbb{A} \subset \mathbb{F}$. For all $q \in Q$ and $\mathbb{A} \subset \mathbb{F}$, we have

$$
\tilde{\gamma}_{i_{1}} \cdots \tilde{\gamma}_{i_{n}}(q \cdot \mathbb{A})=q \cdot\left(\gamma_{i_{1}} \cdots \gamma_{i_{n}}(\mathbb{A})\right) \text {. }
$$

Proposition 4.8 For every $w \in W$, one has

$$
\tilde{\gamma}_{i_{1}} \cdots \tilde{\gamma}_{i_{n}}(E(w))=\bigcap_{\mathcal{U}_{\varepsilon} \in \mathcal{O}, A \in \mathcal{F}} \tilde{\gamma}_{i_{1}} \cdots \tilde{\gamma}_{i_{n}}\left(\mathbb{D}_{\mathcal{U}_{\varepsilon}, A}(w)_{0}\right)
$$

Proof Since $E(w)=\bigcap_{\mathcal{U}_{\varepsilon}, A} \mathbb{D}_{\mathcal{U}_{\varepsilon}, A}(w)_{0}$, it is immediate that

$$
\tilde{\gamma}_{i_{1}} \cdots \tilde{\gamma}_{i_{n}}(E(w)) \subset \bigcap_{\mathcal{U}_{\varepsilon} \in \mathcal{O}, A \in \mathcal{F}} \tilde{\gamma}_{i_{1}} \cdots \tilde{\gamma}_{i_{n}}\left(\mathbb{D}_{\mathcal{U}_{\varepsilon}, A}(w)_{0}\right)
$$

On the other hand, take $\xi=q \cdot v \in \bigcap_{\mathcal{U}_{\varepsilon}, A} \tilde{\gamma}_{i_{1}} \cdots \tilde{\gamma}_{i_{n}}\left(\mathbb{D}_{\mathcal{U}_{\varepsilon}, A}(w)_{0}\right)$. For each pair $\left(\mathcal{U}_{\varepsilon}, A\right) \in \mathcal{O} \times \mathcal{F}$, there is $\xi_{\mathcal{U}_{\varepsilon}, A} \in \mathbb{D}_{\mathcal{U}_{\varepsilon}, A}(w)_{0}$ such that $\xi \in \tilde{\gamma}_{i_{1}} \cdots \tilde{\gamma}_{i_{n}}\left(\xi_{\mathcal{U}_{\varepsilon}, A}\right)$. Note that $\xi_{\mathcal{U}_{\varepsilon}, A} \in \mathbb{E}_{\pi(q)}$ for every $\left(\mathcal{U}_{\varepsilon}, A\right) \in \mathcal{O} \times \mathcal{F}$. Consider the following product direction on $\mathcal{O} \times \mathcal{F}:\left(\mathcal{V}_{\delta}, A\right) \succcurlyeq\left(\mathcal{U}_{\varepsilon}, B\right)$ iff $\mathcal{V}_{\delta}$ refines $\mathcal{U}_{\varepsilon}$ and $A \subset B$. Since $\mathbb{E}_{\pi(q)}$ is compact, we may assume that the net $\left(\xi_{\mathcal{U}_{\varepsilon}, A}\right)$ converges to some point $\eta \in \mathbb{E}_{\pi(q)}$. Note that $\left(\mathcal{V}_{\delta}, A\right) \succcurlyeq\left(\mathcal{U}_{\varepsilon}, B\right)$ implies $\left(\mathcal{S}_{\mathcal{V}_{\delta}, A}\right)_{\mathbb{E}} \zeta \subset\left(\mathcal{S}_{\mathcal{U}_{\varepsilon}, B}\right)_{\mathbb{E}} \zeta$ for any $\zeta \in \mathbb{E}$. Hence, $\mathbb{D}_{\mathcal{V}_{\delta}, A}(w)_{0} \subset \mathbb{D}_{\mathcal{U}_{\varepsilon}, B}(w)_{0}$ whenever $\left(\mathcal{V}_{\delta}, A\right) \succcurlyeq\left(\mathcal{U}_{\varepsilon}, B\right)$. For a given pair $\left(\mathcal{U}_{\varepsilon}, B\right) \in \mathcal{O} \times \mathcal{F}$, it follows that $\left(\xi_{\mathcal{V}_{\delta}, A}\right) \in \mathbb{D}_{\mathcal{V}_{\delta}, A}(w)_{0} \subset \mathbb{D}_{\mathcal{U}_{\varepsilon}, B}(w)_{0}$ for all $\left(\mathcal{V}_{\delta}, A\right) \succcurlyeq\left(\mathcal{U}_{\varepsilon}, B\right)$, and thus $\eta \in \operatorname{cls}\left(\mathbb{D}_{\mathcal{U}_{\varepsilon}, B}(w)_{0}\right)$. Since $E(w)=\bigcap_{\mathcal{U}_{\varepsilon}, A} \operatorname{cls}\left(\mathbb{D}_{\mathcal{U}_{\varepsilon}, A}(w)_{0}\right)$, we have $\eta \in E(w)$, and therefore $\xi \in \tilde{\gamma}_{i_{1}} \cdots \widetilde{\gamma}_{i_{n}}(\eta) \subset \tilde{\gamma}_{i_{1}} \cdots \tilde{\gamma}_{i_{n}}(E(w))$.

Proposition 4.9 The domain of $\mathcal{F}$-chain attraction of $E(w)$ is given by

$$
\mathcal{A}(E(w))=\tilde{\gamma}_{i_{1}} \cdots \tilde{\gamma}_{i_{n}}\left(E\left(w_{0}\right)\right),
$$

where $w_{0} w=r_{i_{n}}, \ldots, r_{i_{1}}$ is a minimal decomposition, and the domain of $\mathcal{F}$-chain repellency of $E(w)$ is given by

$$
\mathcal{A}^{*}(E(w))=\tilde{\gamma}_{j_{1}} \cdots \tilde{\gamma}_{j_{m}}(E(1))
$$

where $w=r_{j_{m}}, \ldots, r_{j_{1}}$ is a minimal decomposition. 
Proof By Theorem 3.2, we have $\mathcal{A}(E(w))=\bigcap_{\mathcal{U}_{\varepsilon}, A} \mathcal{A}\left(\mathbb{D}_{\mathcal{U}_{\varepsilon}, A}(w)\right)$. By [18, Theorem 6.3], we have

$$
\mathcal{A}\left(\mathbb{A}_{\mathcal{U}_{\varepsilon}, A}^{q}(w)\right)=\gamma_{i_{1}} \cdots \gamma_{i_{n}}\left(\mathbb{A}_{\mathcal{U}_{\varepsilon}, A}^{q}\left(w_{0}\right)\right)
$$

for every $q \in Q$, where $w_{0} w=r_{i_{n}}, \ldots, r_{i_{1}}$ is a minimal decomposition. By Lemma 2.2, it follows that

$$
\begin{aligned}
\mathcal{A}(E(w)) \cap \mathbb{E}_{\pi(q)} & =\bigcap_{\mathcal{U}_{\varepsilon}, A} q \cdot \mathcal{A}\left(\mathbb{A}_{\mathcal{U}_{\varepsilon}, A}^{q}(w)\right) \\
& =\bigcap_{\mathcal{U}_{\varepsilon}, A} q \cdot\left(\gamma_{i_{1}} \cdots \gamma_{i_{n}}\left(\mathbb{A}_{\mathcal{U}_{\varepsilon}, A}^{q}\left(w_{0}\right)\right)\right) \\
& =\bigcap_{\mathcal{U}_{\varepsilon}, A} \tilde{\gamma}_{i_{1}} \cdots \tilde{\gamma}_{i_{n}}\left(q \cdot \mathbb{A}_{\mathcal{U}_{\varepsilon}, A}^{q}\left(w_{0}\right)_{0}\right) \\
& =\bigcap_{\mathcal{U}_{\varepsilon}, A} \tilde{\gamma}_{i_{1}} \cdots \tilde{\gamma}_{i_{n}}\left(\mathbb{D}_{\mathcal{U}_{\varepsilon}, A}\left(w_{0}\right)_{0} \cap \mathbb{E}_{\pi(q)}\right)
\end{aligned}
$$

for every $q \in Q$. By Proposition 4.8, we have

$$
\begin{aligned}
\tilde{\gamma}_{i_{1}} \cdots \tilde{\gamma}_{i_{n}}\left(E\left(w_{0}\right)\right) \cap \mathbb{E}_{\pi(q)} & =\bigcap_{\mathcal{U}_{\varepsilon}, A} \tilde{\gamma}_{i_{1}} \cdots \tilde{\gamma}_{i_{n}}\left(\mathbb{D}_{\mathcal{U}_{\varepsilon}, A}\left(w_{0}\right)_{0}\right) \cap \mathbb{E}_{\pi(q)} \\
& =\bigcap_{\mathcal{U}_{\varepsilon}, A} \tilde{\gamma}_{i_{1}} \cdots \tilde{\gamma}_{i_{n}}\left(\mathbb{D}_{\mathcal{U}_{\varepsilon}, A}\left(w_{0}\right)_{0} \cap \mathbb{E}_{\pi(q)}\right) \\
& =\mathcal{A}(E(w)) \cap \mathbb{E}_{\pi(q)}
\end{aligned}
$$

and hence

$$
\mathcal{A}(E(w))=\bigcup_{q \in Q} \mathcal{A}(E(w)) \cap \mathbb{E}_{\pi(q)}=\tilde{\gamma}_{i_{1}} \cdots \tilde{\gamma}_{i_{n}}\left(E\left(w_{0}\right)\right) .
$$

Now, we have

$$
\mathcal{A}^{*}\left(\mathbb{A}_{\mathcal{U}_{\varepsilon}, A}^{q}(w)\right)=\mathcal{A}\left(\mathbb{A}_{\mathcal{U}_{\varepsilon}, A}^{q}\left(w_{0} w\right)^{-}\right),
$$

where $\mathbb{A}_{\mathcal{U}_{\varepsilon}, A}^{q}\left(w_{0} w\right)^{-}$is the effective control set of $\left(S_{\mathcal{U}_{\varepsilon}, A}\right)_{q}^{-1}$ in $\mathbb{F}$. Then, we have

$$
\mathcal{A}^{*}\left(\mathbb{A}_{\mathcal{U}_{\varepsilon}, A}^{q}(w)\right)=\gamma_{j_{1}} \cdots \gamma_{j_{m}}\left(\mathbb{A}_{\mathcal{U}_{\varepsilon}, A}^{q}\left(w_{0}\right)_{0}^{-}\right)=\gamma_{j_{1}} \cdots \gamma_{j_{m}}\left(\mathbb{A}_{\mathcal{U}_{\varepsilon}, A}^{q}(1)_{0}\right)
$$

where $w=w_{0}\left(w_{0} w\right)=r_{j_{m}}, \ldots, r_{j_{1}}$ is a minimal decomposition. Since $\mathcal{A}^{*}(E(w))=$ $\bigcap_{\mathcal{U}_{\varepsilon}, A} \mathcal{A}^{*}\left(\mathbb{D}_{\mathcal{U}_{\varepsilon}, A}(w)\right)$, Proposition 4.8 and Lemma 2.2 together imply that

$$
\begin{aligned}
\mathcal{A}^{*}(E(w)) \cap \mathbb{E}_{\pi(q)} & =\bigcap_{\mathcal{U}_{\varepsilon}, A} q \cdot \mathcal{A}^{*}\left(\mathbb{A}_{\mathcal{U}_{\varepsilon}, A}^{q}(w)\right)=\bigcap_{\mathcal{U}_{\varepsilon}, A} \tilde{\gamma}_{j_{1}} \cdots \tilde{\gamma}_{j_{m}}\left(q \cdot \mathbb{A}_{\mathcal{U}_{\varepsilon}, A}^{q}(1)_{0}\right) \\
& =\bigcap_{\mathcal{U}_{\varepsilon}, A} \tilde{\gamma}_{j_{1}} \cdots \tilde{\gamma}_{j_{m}}\left(\mathbb{D}_{\mathcal{U}_{\varepsilon}, A}(1)_{0} \cap \mathbb{E}_{\pi(q)}\right) \\
& =\tilde{\gamma}_{j_{1}} \cdots \tilde{\gamma}_{j_{m}}\left(E(1) \cap \mathbb{E}_{\pi(q)}\right) .
\end{aligned}
$$

Hence, $\mathcal{A}^{*}(E(w))=\tilde{\gamma}_{j_{1}} \cdots \tilde{\gamma}_{j_{m}}(E(1))$.

We are able to prove the main result of the paper. 
Theorem 4.1 For every $q \in Q, w \in W$, and $\Theta \subset \Sigma$, the intersection of the maximal $\mathcal{F}$-chain transitive set $E_{\Theta}(w)$ with the fiber over $\pi(q)$ is given by

$$
E_{\Theta}(w)_{\pi(q)}=q \cdot \operatorname{fix}_{\Theta}(h(q), w),
$$

where $h(q) \in \operatorname{Ad}(G) H_{\Theta(\sigma)}$ is the point identified with the pair $\left(f(q), f^{*}(q)\right)$.

Proof For $H=\operatorname{Ad}(g) H_{\Theta(\sigma)}$, we have fix $x_{\Theta}(H, w)=g$ fix $_{\Theta}\left(H_{\Theta(\sigma)}, w\right)=g K_{H_{\Theta(\sigma)}}^{0} w b_{\Theta}^{+}$. Since the standard fibration $\pi_{\Theta}: \mathbb{F} \rightarrow \mathbb{F}_{\Theta}$ is equivariant, we have $\pi_{\Theta}($ fix $(H, w))=$ fix $_{\Theta}(H, w)$. In the case of $\Theta(\sigma)$ and $\Theta^{*}(\sigma)$, we have

$$
\begin{gathered}
\text { fix }(H, 1)=\pi_{\Theta(\sigma)}^{-1}\left(\operatorname{fix}_{\Theta(\sigma)}(H, 1)\right), \\
\text { fix }\left(H, w_{0}\right)=\pi_{\Theta^{*}(\sigma)}^{-1}\left(\operatorname{fix}_{\Theta^{*}(\sigma)}\left(H, w_{0}\right)\right),
\end{gathered}
$$

by Corollary 2.1. The components fix $\Theta_{\Theta(\sigma)}(H, 1)$ and $\operatorname{fix}_{\Theta *}(\sigma)\left(H, w_{0}\right)$ reduce to the single points $g b_{\Theta(\sigma)}^{+}$and $g w_{0} b_{\Theta^{*}(\sigma)}^{+}$, respectively. In particular, for a given $q \in Q$, we can write $h(q)=g H_{\Theta(\sigma)}$, and then $h(q g)=H_{\Theta(\sigma)} \sim\left(\mathfrak{p}_{\Theta(\sigma)}, \mathfrak{p}_{\Theta(\sigma)}^{-}\right)$, where $f(q g)=b_{\Theta(\sigma)}^{+}$, that is, $f(q)=g b_{\Theta(\sigma)}^{+}$. Hence, $f(q)=\operatorname{fix}_{\Theta(\sigma)}(h(q), 1)$. Similarly, we can see that $f^{*}(q)=\operatorname{fix}_{\Theta *(\sigma)}\left(h(q), w_{0}\right)$. By Proposition 3.2, it follows that

$$
\begin{aligned}
E(1) \cap \mathbb{E}_{\pi(q)} & =\tilde{\pi}_{\Theta(\sigma)}^{-1}\left(E_{\Theta(\sigma)}(1) \cap\left(\mathbb{E}_{\Theta(\sigma)}\right)_{\pi(q)}\right)=\tilde{\pi}_{\Theta(\sigma)}^{-1}(q \cdot f(q)) \\
& =q \cdot \pi_{\Theta(\sigma)}^{-1}\left(\operatorname{fix}_{\Theta(\sigma)}(h(q), 1)\right) \\
& =q \cdot \operatorname{fix}(h(q), 1)
\end{aligned}
$$

and

$$
\begin{aligned}
E\left(w_{0}\right) \cap \mathbb{E}_{\pi(q)} & =\tilde{\pi}_{\Theta^{*}(\sigma)}^{-1}\left(E_{\Theta^{*}(\sigma)}\left(w_{0}\right) \cap\left(\mathbb{E}_{\Theta^{*}(\sigma)}\right)_{\pi(q)}\right)=\tilde{\pi}_{\Theta^{*}(\sigma)}^{-1}\left(q \cdot f^{*}(q)\right) \\
& =q \cdot \pi_{\Theta^{*}(\sigma)}^{-1}\left(\operatorname{fix}_{\Theta^{*}(\sigma)}\left(h(q), w_{0}\right)\right) \\
& =q \cdot \operatorname{fix}\left(h(q), w_{0}\right) .
\end{aligned}
$$

Now, since $E(w)=\mathcal{A}(E(w)) \cap \mathcal{A}^{*}(E(w))$, Proposition 4.9 implies that

$$
E(w)=\tilde{\gamma}_{i_{1}} \cdots \tilde{\gamma}_{i_{n}}\left(E\left(w_{0}\right)\right) \cap \tilde{\gamma}_{j_{1}} \cdots \tilde{\gamma}_{j_{m}}(E(1))
$$

where $w_{0} w=r_{i_{n}}, \ldots, r_{i_{1}}$ and $w=r_{j_{m}}, \ldots, r_{j_{1}}$ are minimal decompositions. Hence,

$$
\begin{aligned}
E(w) \cap \mathbb{E}_{\pi(q)} & =\tilde{\gamma}_{i_{1}} \cdots \tilde{\gamma}_{i_{n}}\left(E\left(w_{0}\right) \cap \mathbb{E}_{\pi(q)}\right) \cap \tilde{\gamma}_{j_{1}} \cdots \tilde{\gamma}_{j_{m}}\left(E(1) \cap \mathbb{E}_{\pi(q)}\right) \\
& =\tilde{\gamma}_{i_{1}} \cdots \tilde{\gamma}_{i_{n}}\left(q \cdot \operatorname{fix}\left(h(q), w_{0}\right)\right) \cap \tilde{\gamma}_{j_{1}} \cdots \tilde{\gamma}_{j_{m}}(q \cdot \operatorname{fix}(h(q), 1)) \\
& =q \cdot\left(\gamma_{i_{1}} \cdots \gamma_{i_{n}}\left(\operatorname{fix}\left(h(q), w_{0}\right)\right) \cap \gamma_{j_{1}} \cdots \gamma_{j_{m}}(\operatorname{fix}(h(q), 1))\right) .
\end{aligned}
$$

By [3, Corollary 7.5], we have

$$
\text { fix }(h(q), w)=\gamma_{i_{1}} \cdots \gamma_{i_{n}}\left(\operatorname{fix}\left(h(q), w_{0}\right)\right) \cap \gamma_{j_{1}} \cdots \gamma_{j_{m}}(\operatorname{fix}(h(q), 1))
$$

and therefore

$$
E(w) \cap \mathbb{E}_{\pi(q)}=q \cdot \text { fix }(h(q), w) .
$$


Finally, since control sets $\mathbb{A}_{\mathcal{U}_{\varepsilon}, A}\left(w_{0}\right)_{0}$ form a decreasing net of subsets of $\mathbb{F}$, we have

$$
\begin{aligned}
E_{\Theta}(w) \cap\left(\mathbb{E}_{\Theta}\right)_{\pi(q)} & =q \cdot \bigcap_{\mathcal{U}_{\varepsilon}, A} \mathbb{A}_{\mathcal{U}_{\varepsilon}, A}(w)_{0}=q \cdot \bigcap_{\mathcal{U}_{\varepsilon}, A} \pi_{\Theta}\left(\mathbb{A}_{\mathcal{U}_{\varepsilon}, A}(w)_{0}\right) \\
& =q \cdot \pi_{\Theta}\left(\bigcap_{\mathcal{U}_{\varepsilon}, A} \mathbb{A}_{\mathcal{U}_{\varepsilon}, A}\left(w_{0}\right)_{0}\right) \\
& =\tilde{\pi}_{\Theta}\left(E(w) \cap \mathbb{E}_{\pi(q)}\right) \\
& =\tilde{\pi}_{\Theta}(q \cdot \operatorname{fix}(h(q), w)) \\
& =q \cdot \operatorname{fix}(h(q), w) .
\end{aligned}
$$

In particular, we have $\tilde{\pi}_{\Theta}(E(w))=E_{\Theta}(w)$ for all $w \in W$ and $\Theta \subset \Sigma$.

\section{Examples and special cases}

The results of the paper can be applied to fiber bundles with structure group being a reducible Lie group with connected semi-simple component. For instance, $\mathrm{Gl}(n, \mathbb{R})$ is a reductive nonconnected Lie group with semi-simple component $\mathrm{Sl}(n, \mathbb{R})$. The control sets for semigroups in reductive Lie groups are determined only by the action of the semi-simple component; hence, we can develop the results in the semi-simple setting and get for free the same results for reductive groups. See [3, Section 5.3] for details.

\subsection{Vector bundles and Selgrade theorem}

Semigroups of linear isomorphisms on vector bundles are equivalent to semigroup of automorphisms on $\mathrm{Gl}(n, \mathbb{R})$-principal bundles. Then, we can give an alternative proof for the Selgrade theorem about linear actions on vector bundles.

Let $(B, \mathrm{~d})$ be a metric space and $\pi: \mathcal{V} \rightarrow B$ a vector bundle whose typical fiber is $\mathbb{R}^{n}$. Recall that the bundle of frames $\mathrm{B} \mathcal{V} \rightarrow B$ is the locally trivial principal bundle whose elements of $\mathrm{B} \mathcal{V}$ are the invertible linear maps $q: \mathbb{R}^{n} \rightarrow \mathcal{V}_{x}$. The structural group of $\mathrm{B} \mathcal{V}$ is $G=\mathrm{Gl}(n, \mathbb{R})$ which acts on the right on $\mathrm{B} \mathcal{V}$ by $q g=q \circ g, q \in \mathrm{B} \mathcal{V}, g \in \mathrm{Gl}(n, \mathbb{R})$. The vector bundle $\mathcal{V} \rightarrow B$ is recovered from $\mathrm{B} \mathcal{V}$ as the associated bundle $\mathrm{B} \mathcal{V} \times{ }_{\mathrm{Gl}}(n, \mathbb{R}) \mathbb{R}^{n}$ obtained by the standard linear action of $\mathrm{Gl}(n, \mathbb{R})$ in $\mathbb{R}^{n}$.

A subbundle of $\mathcal{V}$ is a closed subset $\mathcal{W} \subset \mathcal{V}$ that intersects each fiber $\mathcal{V}_{x}$ in a linear subspace $\mathcal{W}_{x}$ such that $\operatorname{dim} \mathcal{W}_{x}=\operatorname{dim} \mathcal{W}_{y}$ for all $x, y \in B$. Let $\mathcal{Z} \subset \mathcal{V}$ be the zero section and $\mathcal{X}, \mathcal{Y} \subset \mathcal{V}$ two subbundles with $\mathcal{X} \cap \mathcal{Y} \subset \mathcal{Z}$. The Whitney sum of these subbundles is the vector bundle $\mathcal{W}=\mathcal{X} \oplus \mathcal{Y} \subset \mathcal{V}$ with fibers

$$
\mathcal{W}_{x}=\mathcal{X}_{x} \oplus \mathcal{Y}_{x}=\{\xi+\eta: \xi \in \mathcal{X}, \eta \in \mathcal{Y}\}
$$

Note that $\mathrm{Gl}(n, \mathbb{R})$ is a reducible Lie group with semi-simple component $\mathrm{Sl}(n, \mathbb{R})$, the connected subgroup of matrices with determinant 1 . The corresponding semi-simple Lie algebra is the subalgebra of zero trace matrices $\mathfrak{s l}(n, \mathbb{R})$. The flag manifolds of $\operatorname{Sl}(n, \mathbb{R})$ are the usual manifolds of flags of subspaces of $\mathbb{R}^{n} 1$; hence, the associated flag bundles are the bundles over $B$ which are obtained from $\pi: \mathcal{V} \rightarrow B$ by taking flags of subspaces of $\mathcal{V}_{x}$. 
A Cartan involution $\theta: \mathfrak{s l}(n, \mathbb{R}) \rightarrow \mathfrak{s l}(n, \mathbb{R})$ is given by the automorphism $\theta(X)=-X^{\mathrm{T}}$, whose associated Cartan decomposition is $\mathfrak{s l}(n, \mathbb{R})=\mathfrak{s o}(n, \mathbb{R}) \oplus \mathfrak{s}$, where $\mathfrak{s o}(n, \mathbb{R})$ is the subalgebra of the skew-symmetric matrices and $\mathfrak{s}$ is the subspace of the symmetric matrices. A maximal abelian subalgebra $\mathfrak{a} \subset \mathfrak{s}$ is given by the zero trace diagonal matrices, and a Weyl chamber $\mathfrak{a}^{+} \subset \mathfrak{a}$ is given by the set of zero trace diagonal matrices of the form $H=\operatorname{diag}\left\{a_{1}, \ldots, a_{n}\right\}$ satisfying $a_{1}>\cdots>a_{n}$. Then, $H=\operatorname{diag}\left\{a_{1}, \ldots, a_{n}\right\} \in \operatorname{cls}\left(\mathfrak{a}^{+}\right)$ if and only if $a_{1} \geq \cdots \geq a_{n}$. The adjoint orbit of $H$ is the set of the diagonalizable zero trace matrices with the same eigenvalues as $H$. The set of roots $\Pi$ is given by the linear functionals $\alpha^{i, j}\left(\operatorname{diag}\left\{a_{1}, \ldots, a_{n}\right\}\right)=a_{i}-a_{j}$, with $i \neq j$, and the system of simple roots is the set $\Sigma=\left\{\alpha^{i, i+1}: i=1, \ldots, n-1\right\}$.

For $H=\operatorname{diag}\left\{a_{1}, \ldots, a_{n}\right\}$, we have $\Theta(H)=\left\{\alpha^{i, i+1}: a_{i}=a_{i+1}\right\}$. Then, $\Theta=\Theta(H)$ if and only if $H=\operatorname{diag}\left\{\lambda_{1} I_{d_{1}}, \ldots, \lambda_{k} I_{d_{k}}\right\}$, where $\lambda_{i} I_{d_{i}}$ is a diagonal block, $\lambda_{1}>\cdots>\lambda_{k}$, and

$$
d_{i}=\max \left\{j: \alpha^{c_{i}, c_{i}+1}, \ldots, \alpha^{c_{i}+j, c_{i}+j+1} \in \Theta\right\}
$$

with $c_{1}=0$ and $c_{i}=c_{i-1}+d_{i-1}$.

Now, let $(S, \mathcal{V}, \sigma)$ be a semigroup action that is linear on fibers, that is, $S$ is a semigroup of linear isomorphisms on the vector bundle $\mathcal{V}$. This action lifts to an action on $B \mathcal{V}$ given by $s q=\sigma_{s} \circ q$, which defines a semigroup of automorphisms of $B \mathcal{V}$. Conversely, a semigroup of automorphisms of $B \mathcal{V}$ induces a linear action on the associated bundle $\mathcal{V}$.

Let $\mathcal{F}$ be a filter basis on the subsets of $S$ satisfying the Translation Hypothesis. Assume that the action of $S$ on $B$ is $\mathcal{F}$-chain transitive and the limit sets $\omega(x, \mathcal{F})$ and $\omega^{*}(x, \mathcal{F})$ are nonempty for every $x \in B$. In this case, the maximal $\mathcal{F}$-chain transitive sets $E_{\Theta}(w)(w \in W)$ on a flag bundle coincide with the $\mathcal{F}$-Morse sets of the finest $\mathcal{F}$-Morse decomposition. By Theorem 4.1, $E_{\Theta}(w)$ is given by the set of fixed points of $h(q) \in \operatorname{Ad}(G) H_{\Theta(\sigma)}$, where $h: B \mathcal{V} \rightarrow \operatorname{Ad}(G) H_{\Theta(\sigma)}$ is an equivariant map. For $q \in B \mathcal{V}$ and $x \in B$, we can define the linear map $H_{x}=q \circ h(p) \circ q^{-1}: \mathcal{V}_{x} \rightarrow \mathcal{V}_{x}$. Then, we have the following generalization of Theorem 10.1 in [3].

Theorem 5.1 Let $(S, \mathcal{V}, \mu)$ be a semigroup of linear isomorphisms on the vector bundle $\pi: \mathcal{V} \rightarrow B$, and let $\mathcal{F}$ be a filter basis on the subsets of $S$ satisfying the Translation Hypothesis. Assume that the limit sets $\omega(x, \mathcal{F})$ and $\omega^{*}(x, \mathcal{F})$ are nonempty, for every $x \in B$, and the action of $S$ on $B$ is $\mathcal{F}$-chain transitive. For each $x \in B$, there is a diagonalizable linear map $H_{x}: \mathcal{V}_{x} \rightarrow \mathcal{V}_{x}$ such that the maximal $\mathcal{F}$-chain transitive sets of $S$ in a flag bundle are given fiberwise by the connected components of the set of fixed points of $\exp \left(t H_{x}\right)$. Moreover, the map $x \mapsto H_{x}$ is continuous and the spectra of $H_{x}$ is constant along $B$.

By considering the projective spaces $\mathbb{P}_{x}^{n-1}$ of $\mathcal{V}_{x}$, we have the projective bundle $P \pi$ : $\mathbb{P} \mathcal{V} \rightarrow B$ of $\mathcal{V}$ where $\mathbb{P} \mathcal{V}=\mathrm{B} \mathcal{V} \times{ }_{\mathrm{Gl}}(n, \mathbb{R}) \mathbb{P}^{n-1}$. The fibration $P: \mathcal{V} \backslash \mathcal{Z} \rightarrow \mathbb{P} \mathcal{V}$ is given by $P(q \cdot u)=q \cdot \mathbb{P} u$, where $\mathbb{P}: \mathbb{R}^{n} \backslash\{0\} \rightarrow \mathbb{P}^{n-1}$ denotes the canonical projection map, which satisfies $P \pi \circ P=\pi$. In this special case of flag bundle, the fixed points of $\exp (t h(q))$ in $\mathbb{P}^{n-1}$ are the projections of the eigenvectors $V_{\lambda_{i}}$ of $H_{\Theta(\sigma)}$ associated with the eigenvalues $\lambda_{1}>\cdots>\lambda_{k}$. For each $i=1, \ldots, k$, define $\mathcal{V}_{i}=\left\{q \cdot u \in \mathcal{V}: q \in B \mathcal{V}, u \in V_{\lambda_{i}}\right\}$. Then, $\mathcal{V}_{i}$ is a subbundle of $\mathcal{V},\left\{\mathbb{P V} \mathcal{V}_{i}: i=1, \ldots, k\right\}$ is the collection of the maximal $\mathcal{F}$-chain transitive sets of $S$ on $\mathbb{P V}$, and

$$
\mathcal{V}=\mathcal{V}_{1} \oplus \cdots \oplus \mathcal{V}_{k}
$$

as a Whitney sum. This recovers the Selgrade theorem [24, Theorem 5.1]. 


\subsection{Linearizations}

The study of linearized control systems is one of the motivations for considering semigroup actions on flag bundles.

Let $M$ be an $n$-dimensional manifold and consider a control system $\Sigma$ on $M$ determined by a set of complete vector fields $F$. The system semigroup of $\Sigma$ is the semigroup of diffeomorphisms

$$
S=\left\{\exp \left(t_{1} X_{1}\right) \circ \cdots \circ \exp \left(t_{k} X_{k}\right): X_{i} \in F, \sum_{i=1}^{k} t_{i} \geq 0, t_{i} \geq 0, k \in \mathbb{N}\right\} .
$$

The direction for the limit behavior of $\Sigma$ is usually determined by the filter basis $\mathcal{F}=$ $\left\{S_{\geq T}: T \geq 0\right\}$ where

$$
S_{\geq T}=\left\{\exp \left(t_{1} X_{1}\right) \circ \cdots \circ \exp \left(t_{k} X_{k}\right): X_{i} \in F, \sum_{i=1}^{k} t_{i} \geq T, t_{i} \geq 0, k \in \mathbb{N}\right\} .
$$

In a general case, we consider a semigroup action $(S, M, \sigma)$ such that $\sigma_{S}$ is a diffeomorphism of $M$ for every $s \in S$. The action of $S$ on $M$ lifts to an action of automorphisms on the bundle of frames $\mathrm{B} M$ by defining $s q=d\left(\sigma_{s}\right)_{x} \circ q$, where $d\left(\sigma_{s}\right)_{x}: \mathrm{T}_{x} M \rightarrow \mathrm{T}_{s x} M$ is the derivative of $\sigma_{s}$ and $q: \mathbb{R}^{n} \rightarrow \mathrm{T}_{x} M$ is a frame in $\mathrm{B} M$. This is a special case of linear semigroup action on vector bundle, since the associated bundle $\mathrm{B} M \times \mathrm{Gl}(n, \mathbb{R}) \mathbb{R}^{n}$ recovers the tangent bundle TM of $M$. If $\mathcal{F}$ satisfies the Translation Hypothesis, the action of $S$ on $M$ is $\mathcal{F}$-chain transitive, and the limit sets $\omega(x, \mathcal{F})$ and $\omega^{*}(x, \mathcal{F})$ are nonempty for every $x \in M$, then there is a Whitney sum $\mathrm{T} M=\mathcal{V}_{1} \oplus \cdots \oplus \mathcal{V}_{k}$ such that $\mathbb{P} \mathcal{V}_{1}, \ldots, \mathbb{P} \mathcal{V}_{k}$ are the maximal $\mathcal{F}$-chain transitive sets of $S$ on the projective bundle $\mathbb{P T} M$.

There are interesting cases of diffeomorphisms of $M$ which allow to consider subbundles of $\mathrm{B} M$ and then automorphisms on flag bundles with groups different from $\mathrm{Gl}(n, \mathbb{R})$ (see e.g., [3, Section 10.3]). For instance: (1) A volume preserving semigroup of diffeomorphisms lifts to a semigroup of automorphisms on a $\mathrm{Sl}(n, \mathbb{R})$-flag bundle. (2) A Hamiltonian semigroup of diffeomorphisms preserving a symplectic form lifts to a semigroup of automorphisms on a $\operatorname{Sp}(n, \mathbb{R})$-flag bundle (Lagrangian flag bundle). (3) A semigroup of isometries of a pseudo-Riemannian manifold lifts to automorphisms on a SO $(n, k)$-flag bundle (Lorentzian flag bundle). A semigroup of holomorphic diffeomorphisms of a complex manifold lifts to automorphisms on a $\mathrm{Gl}(n, \mathbb{C})$-flag bundle.

\subsection{Invariant multi-time dynamical systems}

Multi-time dynamical systems appear naturally in $\mathrm{Gl}(n, \mathbb{R})$-bundles and theoretical physics.

An $n$-time dynamical system is a semigroup action $(S, Q, \sigma)$ with $S \subset \mathbb{R}^{n}$ a convex cone. Any linear functional $\lambda: \mathbb{R}^{n} \rightarrow \mathbb{R}$ defines a direction for limit behavior by taking the filter basis $\mathcal{F}$ of the subsets $A_{r}=\{s \in S: \lambda(s) \geq r\}, r \geq 0$. For example, in the Galilean spacetime structure, one has $Q=S=\mathbb{R}^{4}$, the action is given by parallel displacements, and a non-trivial linear functional determines the time axis, which means that the time direction establishes the limit behavior for the action. However, there are further possibilities for limit behavior which are interesting, for instance, the filter basis of the translates $S+s, s \in S$, or the filter basis of the subsets $A_{r}=\{s \in S:\|s\| \geq r\}, r \geq 0$.

A multi-time dynamical system on a principal bundle is called invariant if it determines a semigroup of automorphisms of the bundle. For instance: 1) Let $\pi: Q \rightarrow B$ 
be a principal $\mathrm{Gl}(n, \mathbb{R})$-bundle and take the group homomorphism $\varphi: \mathbb{R}^{n} \rightarrow \mathrm{Gl}(n, \mathbb{R})$ given by $\varphi\left(t_{1}, \ldots, t_{n}\right)=\operatorname{diag}\left\{\mathrm{e}^{t_{1}+\cdots+t_{n}}, \ldots, \mathrm{e}^{t_{1}+\cdots+t_{n}}\right\}$. Define an $n$-time dynamical system $\sigma: \mathbb{R}^{n} \times Q \rightarrow Q$ by

$$
\sigma\left(\left(t_{1}, \ldots, t_{n}\right), q\right)=q \varphi\left(t_{1}, \ldots, t_{n}\right) .
$$

It is easily seen that $\sigma$ is invariant. 2) Let $X_{1}, \ldots, X_{n}$ be pairwise commutative vector fields on a manifold $M$. Define an $n$-time dynamical system $\sigma: \mathbb{R}^{n} \times M \rightarrow M$ by

$$
\sigma\left(\left(t_{1}, \ldots, t_{n}\right), x\right)=\exp \left(t_{1} X_{1}\right) \circ \cdots \circ \exp \left(t_{n} X_{n}\right)(x) .
$$

This is a very special case of control system. As in the example above, $\sigma$ lifts to an invariant $n$-time dynamical system on the bundle of frames $\mathrm{B} M$.

\subsection{Chain recurrence in the fibers}

A semigroup of automorphisms of a principal bundle induces a semigroup action on the fibers of the flag bundle. The chain recurrence in the fibers relates to the chain recurrence on the whole fiber bundle.

We firstly notice that for a given $H \in \operatorname{cls}\left(\mathfrak{a}^{+}\right)$the set $\operatorname{fix}_{\Theta}(H, w)$ is a chain transitive set for the flow $\exp (t H)$ on $\mathbb{F}_{\Theta}$ (Remark 3.3). By Theorem 4.1, the chain transitivity of $\exp (t H)$ on $\mathbb{F}_{\Theta}$ relates to the chain transitivity of the semigroup $S$ on the flag bundle $\mathbb{E}_{\Theta}$. However, under suitable conditions, the semigroup $S$ itself induces a semigroup on the flag $\mathbb{F}_{\Theta}$, whose chain transitivity relates to the chain transitivity of $S$. Indeed, there are situations where the set $A_{q} \subset G$ is nonempty, for every $q \in Q$ and $A \in \mathcal{F}$, where

$$
A_{q}=\{g \in G: \text { there is } s \in A \text { such that } s q=q g\} .
$$

In this case, $\mathcal{F}_{q}=\left\{A_{q}: A \in \mathcal{F}\right\}$ defines a filter basis on the subsets of the subsemigroup $S_{q} \subset G$. The relationship between the $\mathcal{F}$-chain transitivity of $S$ on $\mathbb{E}_{\Theta}$ and the $\mathcal{F}_{q}$-chain transitivity of $S_{q}$ on the fiber $q \cdot \mathbb{F}_{\Theta}$ was extensively studied in $[5,7,8]$.

Since the flag manifold $\mathbb{F}_{\Theta}$ is compact, the chain recurrence on $\mathbb{F}_{\Theta}$ does not depend on the admissible family of open coverings. This is due to the Conley theorem that describes the chain recurrence by means of attractor-repeller pairs [6]. Then, we may consider the family of all open coverings of $\mathbb{F}_{\Theta}$ for chain recurrence. If $E \subset \mathbb{F}_{\Theta}$ is a maximal $\mathcal{F}_{q}$-chain transitive set of $S_{q}$, then $q \cdot E$ is an $\mathcal{F}$-chain transitive set of $S$ in $\mathbb{E}_{\Theta}$ (see proof for Proposition 4.7 in [7]). Hence, $q \cdot E \subset E_{\Theta}(w)_{\pi(q)}=q \cdot \operatorname{fix}_{\Theta}(h(q), w)$ for some $w \in W$, and therefore every maximal $\mathcal{F}_{q}$-chain transitive set of $S_{q}$ consists of fixed points for the flow $\exp (t h(q))$.

If the flag bundle $\mathbb{E}_{\Theta}$ is compact, then the collection $\mathcal{M}_{\Theta}=\left\{E_{\Theta}(w): w \in W\right\}$ of maximal $\mathcal{F}$-chain transitive set is the finest $\mathcal{F}$-Morse decomposition for the action of $S$ on $\mathbb{E}_{\Theta}$ [7, Theorem 5.2]. This means that there is an $\mathcal{F}_{q}$-Morse decomposition $\mathcal{M}_{\Theta}^{q}=$ $\left\{M_{\Theta}^{q}(w): w \in W\right\}$ for the action of $S_{q}$ on $\mathbb{F}_{\Theta}$ such that $q \cdot M_{\Theta}^{q}(w)=E_{\Theta}(w)_{\pi(q)}$ for each $w \in W$ [7, Corollary 5.2]. Hence, $M_{\Theta}^{q}(w)=\operatorname{fix}_{\Theta}(h(q), w)$ for every $w \in W$, and therefore the sets $\operatorname{fix}_{\Theta}(h(q), w), w \in W$, form an $\mathcal{F}_{q}$-Morse decomposition for $S_{q}$ on $\mathbb{F}_{\Theta}$.

\subsection{Direct product semigroup actions}

Semigroups of automorphisms are easily constructed via product actions on trivial bundles. The broad process of construction provides examples of semigroup of automorphisms on flag bundles which need not be differentiable manifolds. 
Let $X$ be any paracompact space and $\Sigma$ a semigroup of homeomorphisms of $X$. Assume that $\mathcal{F}_{\Sigma}$ is a filter basis on the subsets of $\Sigma$ satisfying the Translation Hypothesis, the limit sets are nonempty, and the whole space $X$ is $\mathcal{F}_{\Sigma}$-chain transitive. For instance, let $\Gamma$ be an abelian topological group, $\Sigma \subset \Gamma$ a subsemigroup, and $\mathcal{F}_{\Sigma}$ the family of translates $\Sigma \sigma$, $\sigma \in \Sigma$. The natural action of $\Gamma$ on itself extends to the Stone-Čech compactification $\beta \Gamma$. Define $X=\omega\left(1, \mathcal{F}_{\Sigma}\right) \subset \beta \Gamma$, where 1 is the identity of $\Gamma$. Since $X$ is $S$-invariant, the action of $S$ reduces to a semigroup of homeomorphisms of $X$, the limit sets are nonempty, by compactness, and $X$ is $\mathcal{F}_{\Sigma}$-chain transitive (Remark 3.4).

Let $G$ be a reductive Lie group with connected semi-simple component and $T \subset G$ a subsemigroup. Assume that $\mathcal{F}_{T}$ is a filter basis on the subsets of $T$ satisfying the Translation Hypothesis. For instance, let $T \subset \mathrm{GL}(n, \mathbb{R})$ be the subgroup of the real matrices with positive determinant and $\mathcal{F}_{T}=\left\{A_{r}: r>0\right\}$, where $A_{r}=\{g \in T: \operatorname{det} g>r\}$.

Recall that the direct product of semigroups $S=\Sigma \times T$ has the operation defined componentwise:

$$
\left(\sigma_{1}, t_{1}\right) \cdot\left(\sigma_{2}, t_{2}\right)=\left(\sigma_{1} \sigma_{2}, t_{1} t_{2}\right) .
$$

The direct product action of $S$ on the product space $X \times G$ is given by

$$
(\sigma, t)(x, g)=(\sigma x, \operatorname{tg}) .
$$

This direct product action defines a semigroup of automorphisms on the trivial bundle $X \times$ $G \rightarrow X$. Indeed, we have

$$
(\sigma, t)((x, g) h)=(\sigma, t)(x, g h)=(\sigma x, \operatorname{tgh})=(\sigma x, \operatorname{tg}) h=((\sigma, t)(x, g)) h
$$

for all $(\sigma, t) \in S,(x, g) \in X \times G$, and $h \in G$.

Now, define the family $\mathcal{F}=\left\{A \times B: A \in \mathcal{F}_{\Sigma}, B \in \mathcal{F}_{T}\right\}$. It is not difficult to check that $\mathcal{F}$ is a filter basis on the subsets of $S$ and satisfies the Translation Hypothesis. For any $(x, g) \in X \times G$ and $A \times B \in \mathcal{F}$, we have

$$
\begin{aligned}
(A \times B)_{(x, g)} & =\{h \in G: \text { there is }(\sigma, t) \in A \times B \text { such that }(\sigma, t)(x, g)=(x, g) h\} \\
& =g^{-1} B g
\end{aligned}
$$

and $S_{(x, g)}=g^{-1} T g$. Hence, the filter basis $\mathcal{F}_{(x, g)}=\left\{g^{-1} B g: B \in \mathcal{F}_{T}\right\}$ on $S_{(x, g)}$ depends only on $g$ and $\mathcal{F}_{T}$. Thus, we can write $\mathcal{F}_{(x, g)}=\mathcal{F}_{g}$ for every $x \in X$. Note that $S_{1}=T$ and $\mathcal{F}_{1}=\mathcal{F}_{T}$.

The flag bundle $\mathbb{E}_{\Theta}$ is isomorphic with the trivial bundle $X \times \mathbb{F}_{\Theta}$ by the isomorphism

$$
\begin{aligned}
X \times \mathbb{F}_{\Theta} & \rightarrow \mathbb{E}_{\Theta} \\
(x, u) & \mapsto(x, 1) \cdot u
\end{aligned}
$$

The corresponding action of $S$ on $X \times \mathbb{F}_{\Theta}$ is the direct product action given by $(\sigma, t)(x, u)=$ $(\sigma x, t u)$. For a given $\varepsilon>0$ and an open covering $\mathcal{U}$ of $X$, an adapted covering of $X \times \mathbb{F}_{\Theta}$ is given by

$$
\mathcal{U}_{\varepsilon}=\left\{U \times \mathrm{B}_{\mathrm{d}}(u, \varepsilon): U \in \mathcal{U}, u \in \mathbb{F}_{\Theta}\right\} .
$$

If $(x, u) \in E_{\Theta}(w)$, then $u$ is an $\mathcal{F}_{T}$-chain recurrent point for the action of $T$ on $\mathbb{F}_{\Theta}$ and $E_{\Theta}(w) \subset X \times E_{\Theta}^{1}(w)$, where $E_{\Theta}^{1}(w)$ is the maximal $\mathcal{F}_{T}$-chain transitive set containing $u$. For any $y \in X$, we have seen that $\{y\} \times E_{\Theta}^{1}(w) \subset E_{\Theta}\left(w^{\prime}\right)$, for some $w^{\prime} \in W$. Hence, $E_{\Theta}(w)=E_{\Theta}\left(w^{\prime}\right)$, and therefore

$$
E_{\Theta}(w)=X \times E_{\Theta}^{1}(w), \quad w \in W
$$


Moreover, $\{y\} \times E_{\Theta}^{1}(w)=E_{\Theta}(w)_{y}=\{y\} \times \operatorname{fix}_{\Theta}(h(y, 1), w)$, and hence fix ${ }_{\Theta}(h(y, 1)$, $w)=E_{\Theta}^{1}(w)$ for every $w \in W$.

This is a case in which the chain transitivity on the flag bundle is completely determined by the chain transitivity in the fiber.

\section{References}

1. Alves, R.W.M., Rocha, V.H.L., Souza, J.A.: A characterization of completely regular spaces. Int. J. Math. 26, 1550032 (2015)

2. Borel, A., Tits, J.: Groupes réductifs. Inst. Hautes Études Sci. Publ. Math. 27, 55-152 (1965)

3. Braga Barros, C.J., San Martin, L.A.B.: Chain transitive sets for flows on flag bundles. Forum Math. 19, 19-60 (2007)

4. Braga Barros, C.J., San Martin, L.A.B.: Chain control sets for semigroup actions. Math. Appl. Comput. 15, 257-276 (1996)

5. Braga Barros, C.J., San Martin, L.A.B.: On the action of semigroups in fiber bundles. Mat. Contemp. 13, 1-19 (1997)

6. Braga Barros, C.J., Souza, J.A.: Attractors and chain recurrence for semigroup actions. J. Dyn. Diff. Equ. 22, 723-740 (2010)

7. Braga Barros, C.J., Souza, J.A.: Finest Morse decompositions for semigroup actions on fiber bundles. J. Dyn. Diff. Equ. 22, 741-760 (2010)

8. Braga Barros, C.J., Souza, J.A.: On the number of maximal chain transitive sets in fiber bundles. Forum Math. 25, 363-381 (2013)

9. Clifford, A.H., Preston, G.B.: The Algebraic Theory of Semigroups, vol. II. American Mathematical Society, Providence (1967)

10. Colonius, F., Kliemann, W.: Morse decompositions and spectra on flag bundles. J. Dyn. Diff. Equ. 14, 719-741 (2002)

11. Duistermaat, J.J., Kolk, J.A.C., Varadarajan, V.S.: Functions, flows and oscillatory integrals on flag manifolds and conjugacy classes in real semisimple Lie groups. Compos. Math. 49, 309-398 (1983)

12. Helgason, S.: Differential Geometry, Lie Groups and Symmetric Spaces. Academic, New York (1978)

13. Husemoller, D.: Fibre Bundles. Graduate texts in Mathematics, vol. 20. Springer, New York (1975)

14. Kobayashi, S., Nomizu, K.: Foundations of Differential Geometry. Wiley, New York (1963)

15. Patrão, M.: Semiflows on Flag Bundles and Their Shadowing Semigroups. State University of Campinas, Campinas (2006)

16. Patrão, M., San Martin, L.A.B.: Morse decomposition of semiflows on fiber bundles. Discrete Contin. Dyn. Syst. A 17, 113-139 (2007)

17. San Martin, L.A.B.: Invariant control sets on flag manifolds. Math. Control Sig. Syst. 6, 41-61 (1993)

18. San Martin, L.A.B.: Order and domains of attraction of control sets in flag manifolds. J. Lie Theory 8 , 335-350 (1998)

19. San Martin, L.A.B.: Maximal semigroups in semi-simple Lie groups. Trans. Am. Math. Soc. 353, 51655184 (2001)

20. San Martin, Luiz A.B.: Nonexistence of invariant semigroups in affine symmetric spaces. Math. Ann. 321, 587-600 (2001)

21. San Martin, L.A.B., Tonelli, P.A.: Semigroup actions on homogeneous spaces. Semigroup Forum 50, 59-88 (1995)

22. San Martin, L.A.B., Tonelli, P.A.: Transitive actions of semigroups in semi-simple Lie groups. Semigroup Forum 58, 142-151 (1999)

23. Selgrade, J.: Isolated invariant sets for flows on vector bundles. Trans. Am. Math. Soc. 203, 259-390 (1975)

24. Souza, J.A.: Chain transitivity for semigroup actions on flag bundles. Ann. Mat. Pura Appl. 193, 817-836 (2014)

25. Varadarajan, V.S.: Harmonic Analysis on Real Reductive Groups. Lecture Notes in Math., vol. 576. Springer, Berlin (1977)

26. Warner, G.: Harmonic Analysis on Semi-Simple Lie Groups I. Springer, Berlin (1972) 